\title{
The Role of Outside Options in Auction Design*
}

\author{
Nicolás Figueroa, University of Minnesota ${ }^{\dagger} \quad$ Vasiliki Skreta, UCLA $^{\ddagger}$
}

March 2006

\begin{abstract}
We characterize the revenue maximizing allocation mechanism for a risk neutral seller that owns $N$, possibly heterogeneous, objects and faces $I$ risk neutral buyers with very general preferences, which allow for complements, substitutes and externalities. Buyers care about the entire allocation of the objects, even if they do not win any, so the auction outcome may affect them also when they do not to participate, and these non-participation payoffs may very well depend on their type. The main novel message of our analysis is that with type-dependent non-participation payoffs, the revenue maximizing assignment of objects can crucially depend on the outside options that buyers face. Outside options can therefore affect the degree of efficiency of revenue maximizing auctions. We show that sometimes an optimal mechanism will allocate the objects in an ex-post efficient way, and other times buyers will obtain objects more often than it is efficient. Our characterization rings a bell of caution. Modeling buyers' outside options as being independent of their private information, is with loss of generality and can lead to quite misleading intuitions. Keywords: Optimal Multi Unit Auctions, Type Dependent Outside Options, Externalities, Mechanism Design: JEL D44, Cr, Cr2.
\end{abstract}

\footnotetext{
${ }^{*}$ We are grateful to Masaki Aoyagi, Andrew Atkeson, Sushil Bikhchandani, Hongbin Cai, Harold Cole, Matthias Doepke, Sergei Izmalkov, David Levine, Preston McAfee, Andrew McLennan, Benny Moldovanu, Marcel Richter, and Balazs Szentes for very helpful comments and numerous suggestions. Many thanks to seminar participants at California Institute of Technology, at the Canadian Economic Theory Conference 2005, at the Clarence W. Tow Conference on Auctions and at the Universidad de Chile.

${ }^{\dagger}$ Department of Economics, University of Minnesota, 1035 Heller Hall, 271 19th Ave South, Minneapolis, MN 55455; nicolas@econ.umn.edu;

${ }^{\ddagger}$ Department of Economics, University of California, Los Angeles; Box 951477, Los Angeles, CA 900951477, USA.T3107947241, F310825 9528, skreta@econ.ucla.edu
} 


\section{IntRoduction}

This paper characterizes revenue maximizing allocation mechanisms for multiple objects, allowing for buyers to care about the entire allocation of them, not merely the ones they obtain. Since buyers may care about the final allocation of the objects, even if they do not win any, the auction outcome may affect them also when they do not to participate. Non-participation payoffs may then very well depend on their type. Our formulation hence allows for externalities that can be type and identity dependent. Objects can be heterogeneous, and they can be simultaneously complements for some buyers and substitutes for others. Applications range from the allocation of positions in teams, to the allocation of airport take-off and landing slots, privatization, advertising and many more. Despite its generality the problem is tractable. The main novel message of our analysis is that, with type-dependent non-participation payoffs, the revenue maximizing assignment of objects can crucially depend on the outside options that buyers face. Outside options can therefore affect the degree of efficiency of revenue maximizing auctions. We show that sometimes an optimal mechanism will allocate the objects in an ex-post efficient way. Other times, an optimal mechanism will overallocate objects, in the sense that buyers will obtain objects more often than it is efficient. Our characterization rings a bell of caution. Modeling buyers' outside options as being independent of their private information, is with loss of generality and can lead to quite misleading intuitions. ${ }^{1}$

From the just mentioned results, one can see that our analysis exhibits features that contradict well-known intuitions of monopoly theory. ${ }^{2}$ There, a revenue maximizing monopolist faces a trade-off between revenue maximization and efficiency, and he sacrifices efficiency to increase revenue by restricting the supply below the level that is socially desirable. In contrast, in this paper we have a monopolist that does not always face the trade-off between revenue maximization and efficiency. We show that in some cases the seller can increase both revenue and efficiency by designing appropriate outside options. Moreover, our analysis shows that a revenue maximizing seller will sometimes sell "too much" compared to the socially desirable level. These two new insights are due to the fact that non-participation payoffs can depend on buyers' types.

We now illustrate with a simple example how appropriately chosen outside options can increase the efficiency of revenue maximizing mechanisms. Suppose that a small company in Silicon Valley develops a valuable new technology. This company does not have the necessary infrastructure to reap the benefits of this technology, so it is essentially worthless for it. There is, however, a large firm, (say company A), that is willing to purchase it. From all companies, company A can put the technology to better use, irrespective of its

\footnotetext{
${ }^{1}$ The case of type-independent non-participation payoffs is quite special, because an optimal mechanism will allocate the goods in the same way irrespective of the outside options that buyers face. This allocation is almost always inefficient.

${ }^{2}$ Such a comparison is legitimate since the seller in our model is a multiproduct monopolist who instead of choosing revenue maximizing prices, is choosing revenue maximizing mechanisms.
} 
cost realization, therefore giving the technology to it maximizes the sum of consumer and producer surplus. The value of the new technology to company $\mathrm{A}$ is unknown to the developer, but it is commonly known that it depends on company A's cost parameter and it is given by $500,000-500,000 c$. The developer assesses that the cost $c$ takes equally likely values between 0 and 1 . If company $\mathrm{A}$ does not get the technology and no-one else does either, A's payoff is zero. From the classical papers on optimal auctions, developed by Riley and Samuelson (1981) and Myerson (1981), we know that the best that the developer can do is to make a take-it-or leave-it offer to company A of $\$ 250,000$. Then company A will get the invention only if its cost parameter is below $\frac{1}{2}$. This maximizes ex-ante expected revenue, which is $\$ 125,000$, but of course it is half the time inefficient, because the developer is stuck half the time with a worthless (for it) invention, whereas company A would generate non-negative payoff for all cost realizations.

Now suppose that the developer can make the invention publicly available by making it open source. The payoff of company A in that case depends on its cost parameter and it is given by $100,000-1,000,000 c$. So, if A is very efficient $\left(c<\frac{1}{10}\right)$, it would prefer the invention to become open-source, instead of the seller keeping it, since 100,000-1,000,000c $>0$. If costs are higher than that level, company A fears that smaller firms could be fiercer competitors, so it prefers that the invention does not become available to anyone. If the developer considers threatening company A, in case A drops out of the sale, which threat should it use? The answer is not obvious since the developer does not know company A's cost parameter, so it does not know which alternative "hurts more." ${ }^{3}$ In this paper we show that the optimal threat is to tell A that in the event it does not participate, the seller keeps the invention with probability $\frac{1}{2}$, and makes it open source with probability $\frac{1}{2}$. Faced with this lottery, then company A always participates, and more importantly at the revenue maximizing mechanism is ALWAYS awarded the invention at a price of $\$ 450,000$. Thus, the open source option, even though is never implemented, has an extraordinary effect on the revenue maximizing allocation. It guarantees a higher expected revenue $(\$ 450,000)$, and makes the mechanism efficient. This is one of the main economic messages of this paper: when outside options depend on the buyers' private information, the way the objects will be allocated crucially depends on what outside options buyers face. If the payoff from open sourcing did not depend on $A^{\prime} s$ cost parameter, the seller could have generated more revenue, but the allocation of the invention at the optimal mechanism would be identical and as inefficient, as in the case where open sourcing were not an option. ${ }^{4}$ This example

\footnotetext{
${ }^{3}$ Both of these threats are credible. In case firm $A$ does not participate in the sale, the seller in this example is indifferent between keeping the invention and making it open source. In fact, since there is nothing else the seller can do in that case, both these options are optimal from her perspective and hence credible.

${ }^{4}$ If $A^{\prime} s$ payoff from open sourcing were independent of its type, say it were $-\$ 100,000$, then, from the work of Jehiel, Moldovanu and Stacchetti (1996), JMS (1996), we know that the developer by threatening company A to make the invention open source, can extract payments even if company A does not get the technology, so long as it does not become open source. In this case the optimal auction will have an entry
} 
highlights the crucial role of outside options on the degree of efficiency of revenue maximizing mechanisms.

Apart from these insights, the generality of our model allows for an elegant description of a large number of allocation problems. It is unique because it not only allows for multiple heterogeneous goods, type-dependent outside options and externalities, it also allows for the goods to be simultaneously complements for some buyers and substitutes for others. We now list a few of the potential applications of our model.

- Allocation of rights to a new technology. Our model may offer useful insights on the debate about how new technologies or ideas should be sold. In the example just discussed, we saw the crucial role of the presence of the open source option on the efficiency properties of the revenue maximizing mechanisms: it increased both revenue and efficiency. This is an important area, since the way property rights are assigned on new ideas and technologies does not only affect the way the particular ideas will be implemented in practice, but also the incentives to produce new ones.

- Auctioning of advertisement slots on the internet, TV or radio. Airtime for advertisements on TV and radio is often priced using conventional mechanisms. However, exploiting the presence of externalities is not far from what we already observe in reality. In Germany during the soccer world cup, advertisement slots were sold by category. For instance a slot was allocated only to brewing companies. Then a potential buyer knew a priori that if it did not buy the slot it will go to a competitor. Nowadays, companies like Yahoo! and Google auction-off their advertising slots and are thinking of optimal ways to do so. Our model fits very well many aspects of the problem these companies face: they are selling many advertising slots that can be heterogeneous, some slots may be substitutes and some complements of one another, and clearly buyers care about the slots that their competitors obtain.

- Team Formation. Our model can be used to study a type of procurement auction where the buyer is an organization, (consulting firm, sports team), that wants to hire individuals to perform a task as a team. The compensation that an individual requires depends on who else will join the team. For instance, if individuals joining consist of gurus in the field, someone may consider the experience of working with such people so important, that he may be willing to participate with minimal compensation. On the other hand, if team members are of very poor quality the compensation that he requires may be higher. ${ }^{5}$

Other applications include firm take-overs,${ }^{6}$ allocation of airport take-off and landing

fee of $\$ 100,000$ and then a take-it-or-leave-it offer of $\$ 250,000$. In this case too, company A will get the new technology when it's cost is below $\frac{1}{2}$. Now the expected revenue for the developer will be higher and it will be $\$ 225,000$, but the optimal auction is inefficient, since there is trade only half the time, exactly as in the case without the open sourcing option.

${ }^{5}$ These insights can be useful when one thinks about academic hiring. Clearly academics care a lot about the quality of their colleagues in absolute sense, and also relatively, meaning how good is the match.

${ }^{6}$ Externalities are of huge importance in firm take-overs: Recently (February 2004), Cingular bought AT\&T wireless for $\$ 41$ billion after a bidding war with Vodafone. Some perceive that the big winner of 
slots, and optimal bundling. We finish with a historical application.

- A historical application. The praetorian guard realized the additional benefits of running an auction when negative externalities are present. In the year 196 A.D. they killed the emperor Pertinax and, making a break with "tradition," decided not to hand over the title on someone else for a fixed price, but to run an auction. Historians ${ }^{7}$ cite the fact that there was heavy overbidding, since participants were afraid that in case of not winning the auction, they would be killed by the next emperor, since they would be potential conspirators. This is an example of extreme negative externalities! The experiment was successful from the point of view of the guard, since the auction generated very high revenue, but was not repeated, probably since Didius Iulianus (the winner) lasted only 65 days as emperor and was killed after that, making next bidders reluctant to participate in another auction of this sort.

We now give a brief description of the general setup and the results.

\section{Outline of Setup and Results}

In Section 3 we introduce the model. Our analysis starts in Section 4 by establishing properties of feasible mechanisms, that is mechanisms that satisfy incentive, voluntary participation, and resource constraints. Using properties of feasible mechanisms, we can write the seller's expected revenue as the sum of two terms; a term that weights the virtual surplus of each allocation with the probability that this allocation will prevail for each vector of cost realizations, and a term that is the sum of the payoffs that accrue to the worst type of each buyer. In contrast to the classical work on optimal auctions where virtual surpluses are buyer specific, here they are allocation specific and may depend on the whole vector of types, since every buyer may be affected by an allocation. A more important difference though, is that the payoff that accrues to a buyer at his worst possible type can depend on the assignment rule that the seller wants to implement, whereas in the classical case this term is a constant. This dependence is due to the fact that a buyer's payoff from not participating in the mechanism may depend on his type.

Section 5 characterizes revenue maximizing mechanisms. When the payoff to a bidder's worst type is linear in the allocation rule, the seller's objective function looks a lot like in the classical problem (see Myerson (1981)), but with modified virtual surpluses. The most important difference, is the fact that the modified virtual surplus can be strictly greater than the actual surplus generated from some allocation. This will induce to the seller to sell more than it is efficient. In the 'regular case', that is the case where pointwise optimization leads to an incentive compatible mechanism, the optimal auction assigns probability one to the allocation of objects with the highest virtual surplus. In contrast though, with

this sale will be Verizon even though it was not a participant in the auction (NY Times February 17, 2004 "Verizon Wireless May Benefit From Results of Auction").

${ }^{7}$ This is stated by Edward Gibbon, (1737-94), English historian, in his book "The History of the Decline and Fall of the Roman Empire." 
the classical work on optimal auctions, our problem may fail to be 'regular' even if virtual surpluses are strictly monotonic in a buyer's own type. This new challenge is due to the fact that each buyer cares about more than one allocation, and his payoffs from each of these allocations depend on his type. We provide a sufficient condition for the problem to be regular, which generalizes Myerson's condition. When this condition is violated, one cannot use the standard 'ironing technique,' because incentive compatibility may be violated, even if virtual surpluses are monotonic (and hence trivially 'ironed'). In a companion paper, we illustrate how one proceed in such cases and establish that the revenue maximizing assignment of objects requires randomization between allocations. ${ }^{8}$

Continuing in Section 5, we examine the case where the seller's expected revenue depends nonlinearly in the assignment rule. In this case pointwise optimization cannot be used, but we argue that the problem has enough structure that allows the use of variational methods. There is no general formula that one can obtain for the revenue maximizing assignment rule. Still there are some general properties of the solutions and some techniques that can be useful. ${ }^{9}$ Finally, in Section 6 we present two largely self contained examples. A reader may get a flavor of our findings by looking directly at these examples.

To summarize, our model is tractable despite its generality and has a very large number of potential applications. Moreover, our analysis highlights the importance of carefully chosen outside options. They do not only increase revenue but may also increase efficiency! This issue seems to be known to practitioners, as it is suggested by the design of the UK spectrum auctions,${ }^{10}$ see for instance Klemperer (2004).

\section{Related Literature}

This paper is primarily related to the literature on optimal auction for multiple objects, and to the literature on agency problems with type-dependent outside options. It is also related to the work on mechanism design with externalities and the work on mechanism design with endogenous market structure.

Optimal Multi-Unit Auctions

The literature on optimal multi-unit auctions either models this problem as one where a buyer's type is single dimensional or as one where a buyer's type is a vector. Among others, when private information is single dimensional, Maskin and Riley (1989) analyze the case of unit demands and continuously divisible goods, Gale (1990) the case of discrete goods and supperadditive valuations and, finally, Levin (1997) the case of complements. As in these papers, uncertainty in our model is single dimensional and buyers are risk neutral, but we allow for many goods, (that can be bundled any way the seller likes), multi-unit demands

\footnotetext{
${ }^{8}$ For more information on this point see Figueroa and Skreta (2006b).

${ }^{9}$ More details on that can be found in an earlier version of this paper, Figueroa and Skreta (2005).

${ }^{10}$ We are grateful to Sushil Bikhchandani for pointing this out.
} 
and payoff functions that allow for complements, substitutes and externalities.

Types are multidimensional in Englebrech-Wiggans (1988), who proves revenue equivalence assuming linear payoffs and in Krishna and Perry (2000), who prove it in a more general setting. Armstrong (2000) and Avery and Hendershott (2000) allow for multidimensional uncertainty but because of the difficulties that arise, the analysis only allows two types. Jehiel and Moldovanu (2001) point out that when uncertainty is multidimensional, a revenue maximizing allocation mechanism will allocate the goods inefficiently even if buyers are ex-ante symmetric. ${ }^{11}$ The characterization of the optimum with multi-dimensional types is extremely difficult. Significant progress has been made, but no analytical solution nor general algorithm is known. This paper is less general in the dimensionality of the types, but much more general in all other dimensions.

Agency Problems with Type-dependent Outside Options

Lewis and Sappington (1989) study an agency problem where the outside option of the agent is type-dependent. Among other things, the fact that the critical type is not necessarily the "worst" one mitigates the inefficiencies that arise from contracting under private information. This feature also appears in our analysis. In a similar scenario, Jullien (2000) uses a dual approach to characterize properties of the optimal mechanism such as the possibility of separation, non-stochasticity, etc. In our work we also examine a different set-up with multiple agents, where the mechanism designer can also choose the outside options.

\section{Mechanism Design with Externalities}

Jehiel, Moldovanu and Stacchetti, (JMS) (1996) study optimal auction with typeindependent externalities in a single unit environment. ${ }^{12}$ Aseff and Chade (2003) analyze a problem where there are two units, buyers demand a single unit, their payoffs are linear in types, and externalities are identity-, but not type-dependent. Brocas (2005) examines a scenario with one good, two buyers with linear payoffs and type-dependent externalities. JMS (1999) consider the design of optimal auctions of a single unit in the presence of type-dependent externalities. The type of each buyer is a vector, where each component indicates his/her utility as a function of who gets the object. The multi-dimensionality of types makes the solution of the general problem intractable.

Optimal Auction Design with Endogenous Market Structure

Our model captures scenarios of auctions with endogenous market structure and generalizes previous work by Dana and Spier (1994), and Milgrom (1996). ${ }^{13}$

\footnotetext{
${ }^{11}$ These observations are provided without a complete characterization of the optimum.

${ }^{12}$ General models allowing for type dependent externalities like those of Jehiel-Moldovanu (2001b), and Krishna and Perry (2000) are concerned with the design of efficient mechanisms.

${ }^{13}$ Gale (1990) also considers a variation of this problem but because he imposes a very strong superadditivity condition to the profit function, he shows that an optimal mechanism always gives all the "permits" to at most one buyer, so the market structure is always a monopoly.
} 


\section{THE MODEL}

A risk neutral seller owns $N$ indivisible, possibly heterogeneous, objects that are of 0 value to her and faces $I$ risk-neutral buyers. Both $N$ and $I$ are finite natural numbers. The seller (indexed by zero) can bundle these $N$ objects in any way she sees fit. An allocation $z$ is an assignment of objects to the buyers and to the seller. It is a vector with $N$ components, where each component stands for an object and it specifies who gets it, therefore the set of possible allocations is finite and given by $Z \subseteq[I \cup\{0\}]^{N}$. Buyer $i$ 's valuation from allocation $z$ is denoted by $\pi_{i}^{z}\left(c_{i}, c_{-i}\right)$ and it depends on buyer $i^{\prime} s$ cost parameter $c_{i}$ and on the cost parameters of all the other buyers $c_{-i}$. Values are therefore interdependent. Buyer $i$ 's cost parameter $c_{i}$ is private information and is distributed on $C_{i}=\left[\underline{c_{i}}, \overline{c_{i}}\right]$, with $0 \leq \underline{c_{i}} \leq \overline{c_{i}}<\infty$, according to a distribution $F_{i}$ that has a strictly positive and continuous density $f_{i}$. All buyers' types are independently distributed. We use $f(c)=\times_{i \in I} f_{i}\left(c_{i}\right)$, where $c \in C=\times_{i \in I} C_{i}$ and $f_{-i}\left(c_{-i}\right)=\times_{\substack{j \in I \\ j \neq i}} f_{j}\left(c_{j}\right)$.

We assume that, for all $i \in I, \pi_{i}^{z}\left(\cdot, c_{-i}\right)$ is decreasing, convex and differentiable for all $z$ and $c_{-i}$. We impose no restrictions on how $\pi_{i}$ depends on $z$ nor $c_{-i}$. This formulation allows for buyers to be demanding many objects, which may be complements or substitutes, and for externalities. It is very well possible that $\pi_{i}^{z}\left(c_{i}, c_{-i}\right) \neq 0$ even when the allocation $z$ does not include any objects for $i$. An instance of that is a situation where buyers are firms competing in different markets, and whatever happens in the current sale will affect their positioning and interaction relative to the other buyers in other markets. More importantly, an allocation may affect buyer $i$ even if he is not taking part in the auction, which implies that non-participation payoffs may depend on $i^{\prime} s$ type. Type-dependent non-participation payoffs are the key force behind our new insights.

The objective of the seller is to design a mechanism that maximizes expected revenue, and buyers aim to maximize expected surplus.

\section{Mechanisms}

By the revelation principle we know that, for a given set of participating buyers, the seller can without loss of generality restrict attention to incentive compatible direct revelation mechanisms.

A direct revelation mechanism, (DRM), $M=(p, x)$ consists of an assignment rule $p: C \longrightarrow \Delta(Z)$ and a payment rule $x: C \longrightarrow \mathbb{R}^{I}$.

The assignment rule specifies the probability of each allocation for a given vector of reports. We denote by $p^{z}(c)$ the probability that allocation $z$ is implemented when the vector of reports is $c$. Observe that the assignment rule has as many components as the number of possible allocations. The payment rule $x$ specifies, for each vector of reports $c$, a vector of payments, one for each buyer. We now proceed to describe the seller's and the buyers' payoffs. 


\section{Payoffs from Participation}

The interim expected utility of a buyer of type $c_{i}$ when he participates and declares $c_{i}^{\prime}$ is

$$
U_{i}\left(c_{i}, c_{i}^{\prime} ;(p, x)\right)=E_{c_{-i}}\left[\sum_{z \in Z}\left(p^{z}\left(c_{i}^{\prime}, c_{-i}\right) \pi_{i}^{z}\left(c_{i}, c_{-i}\right)\right)-x_{i}\left(c_{i}^{\prime}, c_{-i}\right)\right] .
$$

\section{Payoffs from Non-Participation - Non-Participation Assignment Rules}

The payoff that accrues to buyer $i$ from non participation depends on what allocations that will prevail in that case, and on his type $c_{i}$. We denote by $Z^{-i}$ the set of allocations that are feasible ${ }^{14}$ when buyer $i$ refuses to participate. The assignment rule that prevails is denoted by $p^{-i}$ and it maps $C_{-i}$ to $\Delta\left(Z^{-i}\right)$. In this case $i^{\prime}$ 's payoff is given by

$$
\underline{U}_{i}\left(c_{i}, p^{-i}\right)=E_{c_{-i}}\left[\sum_{z \in Z^{-i}}\left(p^{-i}\right)^{z}\left(c_{-i}\right) \pi_{i}^{z}\left(c_{i}, c_{-i}\right)\right],
$$

where $\left(p^{-i}\right)^{z}$ denotes the probability assigned to allocation $z$ by $p^{-i}$. The fact that $i^{\prime} s$ non-participation payoffs depend on his type is probably the most crucial feature of our model.

A non-participation assignment rule specifies a $p^{-i}$ for each $i \in I$. The seller chooses $p^{-i}$ out of the set of admissible non-participation assignment rules which we call $\mathcal{P}^{-i}$. If $p^{-i}$ is exogenously given, as in most of the mechanism design literature, then $\mathcal{P}^{-i}$ is a singleton. If the seller can choose $p^{-i}$, then the elements of $\mathcal{P}^{-i}$ depend on her commitment power. If the seller can commit ex-ante on $p^{-i}$, then $\mathcal{P}^{-i}$ contains all the assignment rules that are possible when $i$ is not around, namely $\mathcal{P}^{-i}=\left\{p^{-i}: C_{-i} \rightarrow \Delta\left(Z^{-i}\right)\right\}{ }^{15}$ If the seller does not have such commitment power, then $\mathcal{P}^{-i}$ contains all the assignment rules that are feasible and optimal when $i$ is not around. It is worthwhile stressing that the new insights we obtain do not hinge upon how non-participation payoffs are determined.

We proceed to describe the timing.

\section{Timing}

Stage 0: The seller chooses a mechanism $(p, x)$ and $p^{-i}$, for all $i$

Stage 1: Buyers decide whether to participate or not. If all participate, they report the types and the mechanism determines the assignment of objects and the payments. If buyer $i$ decides not to participate, the objects are assigned according to $\left\{p^{-i}\right\}$. If more than one buyers fail to participate, we assume that the seller keeps the objects.

\footnotetext{
${ }^{14}$ For example in the case that the seller cannot force buyer $i$ to get objects, $Z^{-i} \subset Z$ is the subset of allocations where $i$ does not obtain any objects.

${ }^{15}$ An instance of this scenario is used in JMS (1996), where there is one object and type independent outside options.
} 
It is also easy to see that it is without loss of generality to assume that all buyers participate for all realizations of their types. The seller can easily replicate an assignment of the objects when buyer $i$ is not participating, by simply having $i$ participate and giving him no objects. ${ }^{16}$

We think of this capturing a one-shot scenario. Given that others participate and tell the truth about their types, is it a best response for buyer $i$ to participate and tell the truth about his type? In such a one-shot scenario, buyers are not making inferences about the types of buyer $i$ in the event that buyer $i$ does not participate.

We now provide a formal definition of what it entails for a direct revelation mechanism to be feasible.

\section{Feasible Mechanisms}

Definition 1. (Feasible Mechanisms) For a given non-participation assignment rule, $\left(p^{-i}\right)_{i \in I}$, we say that a mechanism $(p, x)$ is feasible iff it satisfies

(IC) "incentive constraints," a buyer's strategy is such that $U_{i}\left(c_{i}, c_{i} ;(p, x)\right) \geq U_{i}\left(c_{i}, c_{i}^{\prime} ;(p, x)\right)$ for all $c_{i}, c_{i}^{\prime} \in C_{i}$, and $i \in I$

$(P C)$ "voluntary participation constraints," $U_{i}\left(c_{i}, c_{i} ;(p, x)\right) \geq \underline{U}_{i}\left(c_{i}, p^{-i}\right)$ for all $c_{i} \in C_{i}$, and $i \in I$

$(R E S)$ "resource constraints" $\sum_{z \in Z} p^{z}(c)=1, p^{z}(c) \geq 0$ for all $c \in C$

Summarizing, feasibility requires that $p$ and $x$ are such that buyers (1) prefer to tell the truth about their cost parameter, (2) buyers choose voluntarily to participate in the mechanism and (3) $p$ is a probability distribution over $Z .^{17}$

We now state the seller's problem.

\section{The Seller's Problem}

With the help of the revelation principle the seller's problem can be written as

$$
\begin{aligned}
& \max \int_{C} \sum_{i=1}^{I} x_{i}(c) f(c) d c \\
& \text { subject to }(p, x) \text { being "feasible." }
\end{aligned}
$$

This completes the description of our model and the seller's problem. We proceed with the analysis of it. Proofs of the results not presented in the main text can be found in the Appendix.

\footnotetext{
${ }^{16}$ Here we implicitly assume that the set of possible allocations $Z$ is larger when more buyers participate. This is automatically satisfied when $Z=\{I \cup\{0\}\}^{N}$.

${ }^{17}$ Notice that $Z$ contains the allocation where the seller keeps all the objects, thus $\sum_{z \in Z} p^{z}(c)=1$.
} 


\section{Analysis of the Problem}

This section contains the main analysis of the problem. The seller's objective is to maximize expected revenue subject to incentive, participation and resource constraints. We start by investigating the implications of these constraints.

\section{Implications of Incentive Compatibility}

Given a $\operatorname{DRM}(p, x)$ buyer $i^{\prime} s$ maximized payoff,

$$
V_{i}\left(c_{i}\right)=\max _{c_{i}^{\prime}} \int_{C_{-i}}\left(\sum_{z \in Z} p^{z}\left(c_{i}^{\prime}, c_{-i}\right) \pi_{i}^{z}\left(c_{i}, c_{-i}\right)-x_{i}\left(c_{i}^{\prime}, c_{-i}\right)\right) f_{-i}\left(c_{-i}\right) d c_{-i},
$$

is convex, since it is a maximum of convex functions. In the next Lemma we show that the incentive constraints translate into the requirement that the derivative of $V_{i}$

$$
P_{i}\left(c_{i}\right) \equiv \int_{C_{-i}} \sum_{z \in Z} p^{z}\left(c_{i}, c_{-i}\right) \frac{\partial \pi_{i}^{z}\left(c_{i}, c_{-i}\right)}{\partial c_{i}} f_{-i}\left(c_{-i}\right) d c_{-i},
$$

(more precisely a selection from its subgradient, which is single valued almost surely), evaluated at the true type is weakly increasing. ${ }^{18}$ See Figure 1.

\section{INSERT FIGURE 1 HERE.}

Lemma 1 For a given non-participation assignment rule, $\left(p^{-i}\right)_{i \in I}$, a mechanism $(p, x)$ is incentive compatible iff

$$
\begin{array}{cc}
P_{i}\left(c_{i}^{\prime}\right) \geq P_{i}\left(c_{i}\right) & \text { for all } c_{i}^{\prime}>c_{i} \\
V_{i}\left(c_{i}\right)=V_{i}\left(\bar{c}_{i} ; p, p^{-i}\right)-\int_{c_{i}}^{\overline{c_{i}}} P_{i}(s) d s & \text { for all } c_{i} \in C_{i}
\end{array}
$$

With the help of Lemma 1 and using standard arguments, we can write buyer $i$ 's expected payment as a function of the assignment rule $p$, and the payoff that accrues to his worst type, ${ }^{19} V_{i}\left(\bar{c}_{i} ; p, p^{-i}\right)$

$$
\int_{C} x_{i}(c) f(c) d c=\int_{C} \sum_{z \in Z} p^{z}\left(c_{i}, c_{-i}\right)\left(\pi_{i}^{z}\left(c_{i}, c_{-i}\right)+\frac{F_{i}\left(c_{i}\right)}{f_{i}\left(c_{i}\right)} \frac{\partial \pi_{i}^{z}\left(c_{i}, c_{-i}\right)}{\partial c_{i}}\right) f(c) d c-V_{i}\left(\bar{c}_{i} ; p, p^{-i}\right) .
$$

Let

$$
J_{z}(c) \equiv \sum_{i=1}^{I}\left[\pi_{i}^{z}\left(c_{i}, c_{-i}\right)+\frac{F_{i}\left(c_{i}\right)}{f_{i}\left(c_{i}\right)} \frac{\partial \pi_{i}^{z}\left(c_{i}, c_{-i}\right)}{\partial c_{i}}\right]
$$

\footnotetext{
${ }^{18}$ In the classical case, where there is only one object and $i^{\prime} s$ payoff from obtaining the object is $v_{i}$, (see Myerson (1981)), the analog of $P_{i}$ is $P_{i}\left(v_{i}\right)=\int_{V_{-i}} p\left(v_{i}, v_{-i}\right) f_{-i}\left(v_{-i}\right) d v_{-i}$.

${ }^{19}$ For more details see Appendix.
} 
denote the virtual surplus of allocation $z$. Notice that we are summing over all buyers because an allocation may affect all of them, and not just the ones that obtain objects. Therefore the virtual surplus of allocation $z$ may depend on the whole vector of types. ${ }^{20}$

Using this definition the seller's objective function can be rewritten as

$$
\int_{C} \sum_{z \in Z} p^{z}(c) J_{z}(c) f(c) d c-\sum_{i=1}^{I} V_{i}\left(\bar{c}_{i} ; p, p^{-i}\right) .
$$

Now we turn to examine the implications of the participation constraints.

\section{Implications of Participation Constraints}

Since the seller's revenue is decreasing in $V_{i}\left(\bar{c}_{i} ; p, p^{-i}\right)$, at a solution she will make this constant as small as possible subject to the participation constraint $V_{i}\left(c_{i}\right) \geq \underline{U}_{i}\left(c_{i}, p^{-i}\right)$ for all $c_{i} \in C_{i}$.

These observations imply that there will be at least one type $c_{i}$ where $V_{i}\left(c_{i}\right)=\underline{U}_{i}\left(c_{i}, p^{-i}\right)$.

We call this the critical type of $i$ and denote it by $c_{i}^{*}\left(p, p^{-i}\right)$. Recalling that $V_{i}\left(c_{i}\right)=$ constant $\left.-\int_{c_{i}}^{\overline{c_{i}}} P_{i}(s) d s\right)$, we get that

$$
c_{i}^{*}\left(p, p^{-i}\right) \in \arg \min _{c_{i}}\left[-\int_{c_{i}}^{\bar{c}_{i}} P_{i}(s) d s-\underline{U}_{i}\left(c_{i}, p^{-i}\right)\right] .
$$

See Figure 2. INSERT Figure 2 Here.

At $c_{i}^{*}$ we have that $V_{i}\left(c_{i}^{*}\right)=\underline{U}_{i}\left(c_{i}^{*}, p^{-i}\right)$ and from a generalization of the Fundamental Theorem of Calculus (see Krishna and Maenner (2001)), and incentive compatibility it follows that $V_{i}\left(\bar{c}_{i} ; p, p^{-i}\right)=V_{i}\left(c_{i}^{*}\right)+\int_{c_{i}^{*}}^{\overline{c_{i}}} P_{i}(s) d s$ or

$$
V_{i}\left(\bar{c}_{i} ; p, p^{-i}\right)=\underline{U}_{i}\left(c_{i}^{*}\left(p, p^{-i}\right), p^{-i}\right)+\int_{c_{i}^{*}\left(p, p^{-i}\right)}^{\overline{c_{i}}} P_{i}(s) d s .
$$

From (6) we see that $V_{i}\left(\bar{c}_{i} ; p, p^{-i}\right)$ depends on $p$ through two channels: $P_{i}$ and $c_{i}^{*}\left(p, p^{-i}\right)$.

Moreover, as already discussed, $p^{-i}$ is often not exogenous, and can be chosen by the seller in order to minimize $V_{i}\left(\bar{c}_{i} ; p, p^{-i}\right)$, namely

$$
p^{-i}(p) \in \arg \min _{\rho^{-i} \in \mathcal{P}^{-i}} \underline{U}_{i}\left(c_{i}^{*}\left(p, \rho^{-i}\right), \rho^{-i}\right)+\int_{c_{i}^{*}\left(p, \rho^{-i}\right)}^{\overline{c_{i}}} P_{i}(s) d s .
$$

\footnotetext{
${ }^{20}$ In the classical case virtual valuations are buyer-specific. For buyer $i$ we have $J_{i}\left(v_{i}\right)=v_{i}-\frac{1-F_{i}\left(v_{i}\right)}{f_{i}\left(v_{i}\right)},\left(v_{i}\right.$ is $i$ 's valuation for the object).
} 
For each potential assignment of the objects $p$, there is a potentially different optimal "threat" $p^{-i}(p)$, which can be random. The choice set of $p^{-i}$, namely $\mathcal{P}^{-i}$, depends on the seller's commitment power. ${ }^{21}$ The dependence of $p^{-i}$ on $p$ adds an additional level of complication.

Summarizing, incentive compatibility and participation constraints imply that an optimal mechanism maximizes (4), with $c_{i}^{*}\left(p, p^{-i}\right)$ given by $(5) ; V_{i}\left(\bar{c}_{i} ; p, p^{-i}\right)$ given by $(6)$; and $p^{-i}(p)$ given by $(7)$. In the next proposition we use these properties to restate the seller's problem.

\section{The Seller's Problem}

Proposition 2 If in a mechanism $(\hat{p}, \hat{x})$ the assignment function $\hat{p}$ solves:

$$
\begin{array}{ll}
\max _{p} & \int_{C} \sum_{z \in Z} p^{z}(c) J_{z}(c) f(c) d c-\sum_{i=1}^{I} V_{i}\left(\bar{c}_{i} ; p, p^{-i}(p)\right) \\
\text { s.t. } & P_{i} \text { increasing, } \sum_{z \in Z} p^{z}(c)=1 \text { and } p(c) \geq 0
\end{array}
$$

where $c_{i}^{*}\left(p, p^{-i}\right)$ is given by (5); $V_{i}\left(\bar{c}_{i} ; p, p^{-i}\right)$ is given by $(6) ; p^{-i}(p)$ is given by (7), and the payment function $\hat{x}$ for all $i$ is given by:

$$
\hat{x}_{i}(c)=\sum_{z \in Z} \hat{p}^{z}(c) \pi_{i}^{z}(c)+\int_{c_{i}}^{\overline{c_{i}}} \sum_{z \in Z} \frac{\partial \pi_{i}^{z}\left(s, c_{-i}\right)}{\partial s} \hat{p}^{z}\left(s, c_{-i}\right) d s-V_{i}\left(\bar{c}_{i} ; \hat{p}, p^{-i}\right),
$$

then, the mechanism is optimal.

This program has a structure similar to the standard formulation of the optimal auction problem. For example, we have revenue equivalence. Any two mechanisms that allocate the objects in the same way and give the same expected payoff to the worst type, generate the same revenue. There are however, important differences.

In the standard case where non-participation payoffs are independent of types, (see Myerson (1981) or JMS (1996)), the term $V_{i}\left(\bar{c}_{i} ; p, p^{-i}(p)\right)$ is independent of the assignment rule $p$, because $c_{i}^{*}$ is always equal to $\bar{c}_{i}$. There, the seller's objective function is linear in the assignment rule $p$. The revenue maximizing $p$ is independent of the outside options that buyers face, and it has a simple characterization. In our problem though, $V_{i}\left(\bar{c}_{i} ; p, p^{-i}(p)\right)$ depends on $p$, and that changes the qualitative features of the solution. Also, an optimal $p$ depends on the outside options that buyers face and, for different outside options, (which determine $c_{i}^{*}$, the critical type), the efficiency properties of revenue maximizing assignment rules will differ.

\footnotetext{
${ }^{21}$ It may be worth stressing, that the main force of our results is the fact that outside options are type dependent, and not on whether the seller has the power to choose $p^{-i}$ or not. In most of the mechanism design literature outside options are exogenous. There $\mathcal{P}^{-i}$ is a singleton.
} 
It is possible that $V_{i}\left(\bar{c}_{i} ; p, p^{-i}(p)\right)$ depends non-linearly on $p$, which would imply that the seller's objective function is a non-linear function of $p$. In that case, it is not possible in general to say much about the solution. Its shape and features depend on the particular details of the problem at hand, namely on the shape of the payoff and distribution functions. When $V_{i}\left(\bar{c}_{i} ; p, p^{-i}(p)\right)$ is linear in $p$, then there is a simple analytical characterization of the solution. Given that there is not much hope to say something general in the case that the problem is nonlinear, and given that the solution in this case is likely to not have a simple analytical form, we focus on the cases where $V_{i}\left(\bar{c}_{i} ; p, p^{-i}(p)\right)$ is linear in $p$. This case has an analytical characterization and allows us to see the new economic intuitions that arise from type dependent outside options clearly. First we provide conditions for this to be true, which are satisfied by many environments. Then, we proceed with the characterization of optimal mechanisms. Details of the solution, as well as the formal statements of these observations are in the following section.

\section{Optimal Mechanisms}

We start by examining cases that have simple characterizations, which amount to the cases where the term $V_{i}\left(\bar{c}_{i} ; p, p^{-i}(p)\right)$ is linear in $p$. Then we examine what happens in the case of nonlinearities.

\subsection{Optimal Mechanisms when $V_{i}\left(\bar{c}_{i} ; p, p^{-i}(p)\right)$ is linear in $p$}

When $V_{i}\left(\bar{c}_{i} ; p, p^{-i}(p)\right)$ is linear in $p$, the seller's objective function is linear and the solution simplifies. The main instance of this is when despite the fact that $p^{-i}$ can depend on $p$, neither $c_{i}^{*}\left(p, p^{-i}(p)\right)$ nor the level of $\underline{U}_{i}\left(., p^{-i}(p)\right)$ evaluated at the critical type $c_{i}^{*}$, depend on $p$. This means that for every possible assignment rule $p$, when the seller chooses $p^{-i} \in \mathcal{P}^{-i}$ optimally, that is according to (7), the following are true ${ }^{22}$

$$
\begin{aligned}
c_{i}^{*} & \equiv c_{i}^{*}\left(p, p^{-i}(p)\right) \text { and } \\
\underline{U}_{i}\left(c_{i}^{*}\right) & \equiv \underline{U}_{i}\left(c_{i}^{*}, p^{-i}(p)\right) .
\end{aligned}
$$

There are many instances that satisfy (9) with $c_{i}^{*}=\underline{c}_{i}$, or $c_{i}^{*}=\bar{c}_{i}$; or $c_{i}^{*} \in\left(\underline{c}_{i}, \bar{c}_{i}\right)$ and we now describe a few. These cases are suggestive, and the list is not, nor it is meant to be, exhaustive. A reader not interested in such details, may skip them and start reading after Proposition 6 .

One well known environment that satisfies (9), is the case of type independent, "flat" outside options. In that case the participation constraint always binds at the worst type $c_{i}^{*}=\bar{c}_{i}$. The other extreme, namely when outside options are very responsive to type, exhibits in some sense the reverse phenomenon, and the participation constraint always

\footnotetext{
${ }^{22}$ Notice that if $p^{-i}$ is exogenous $\left(\mathcal{P}^{-i}\right.$ is a singleton) the second requirement is trivially satisfied
} 
binds at the best type $c_{i}^{*}=\underline{c}_{i}$. We now provide the precise conditions for the case of "very responsive" outside options, and argue that under those conditions (9) are satisfied at $c_{i}^{*}=\underline{c}_{i}$.

Let $\bar{\pi}_{i}^{z}\left(c_{i}\right)=\int_{C_{-i}} \pi_{i}^{z}\left(c_{i}, c_{-i}\right) f_{-i}\left(c_{-i}\right) d c_{-i}$ be the expected payoff to agent $i$ if allocation $z$ is implemented.

Assumption 3 Suppose that outside options are steep, in the sense that for all $i \in I$, there exists an allocation $z_{i}^{*} \in Z^{-i}$ such that

$$
\frac{d \bar{\pi}_{i}^{z_{i}^{*}}\left(c_{i}\right)}{d c_{i}} \leq \frac{d \bar{\pi}_{i}^{z}\left(c_{i}\right)}{d c_{i}} \text { for all } z \in Z
$$

and

$$
\bar{\pi}_{i}^{z_{i}^{*}}\left(\underline{c}_{i}\right) \leq \bar{\pi}_{i}^{z}\left(\underline{c}_{i}\right) \text { for all } z \in Z
$$

Proposition 4 Under Assumption 3, and if the rule $p^{-i}$ defined by $\left(\hat{p}^{-i}\right)^{z} \equiv \begin{cases}1 & \text { if } z=z_{i}^{*} \\ 0 & \text { if not }\end{cases}$ belongs to $\mathcal{P}^{-i}$, then $c_{i}^{*}=\underline{c}_{i}$, for all $i$.

Another interesting case is roughly a mixture of the previous two. The seller can choose between a very flat and a very responsive to type outside option. There the participation constraint binds at an interior type $c_{i}^{*} \in\left(\underline{c}_{i}, \bar{c}_{i}\right)$. Suppose that there are two extreme allocations for each buyer, one that gives the flattest payoff $z_{1}^{i}$, and one that gives the steepest, $z_{2}^{i}$. If the flattest option were to be used then $c_{i}^{*}=\bar{c}_{i}$ and if the steepest option were to be used, then $c_{i}^{*}=\underline{c}_{i}$. It turns out that an optimal $p^{-i}$ randomizes between the two options and the participation constraint always binds at the type who is indifferent between $z_{1}^{i}$ and $z_{2}^{i}$. We know describe the precise conditions and establish the claim.

Assumption 5 Suppose that $Z^{-i}=\left\{z_{1}^{i}, z_{2}^{i}\right\}$ and that $\bar{\pi}_{i}^{z_{1}^{i}}\left(c_{i}\right), \bar{\pi}_{i}^{z_{2}^{i}}\left(c_{i}\right)$ satisfy $\frac{d \bar{\pi}_{i}^{i}\left(c_{i}\right)}{d c_{i}} \leq$ $\frac{d \bar{\pi}_{i}^{z}\left(c_{i}\right)}{d c_{i}} \leq \frac{d \bar{\pi}_{i}^{z_{2}^{i}}\left(c_{i}\right)}{d c_{i}}$ for all $z \in Z$ and $c_{i} \in C_{i}$ and $\bar{\pi}_{i}^{z_{1}^{i}}\left(\underline{c}_{i}\right) \geq \bar{\pi}_{i}^{z_{2}^{i}}\left(\underline{c}_{i}\right)$. Suppose also that the seller can only use non-participation assignment rules that do not depend on the types of other players (that is $p^{-i} \in \mathcal{P}^{-i} \Longrightarrow p^{-i}\left(c_{-i}\right) \equiv p^{-i}$ ).

Proposition 6 Under Assumption 5, and if the rule $p^{-i}(p)$ defined by

$$
\left(\hat{p}^{-i}\right)^{z} \equiv \begin{cases}p & \text { if } z=z_{1}^{i} \\ 1-p & \text { if } z=z_{2}^{i} \\ 0 & \text { if not }\end{cases}
$$

belongs to $\mathcal{P}^{-i}$ for all $p \in(0,1)$, then $c_{i}^{*} \in\left(\underline{c}_{i}, \bar{c}_{i}\right)$, where $c_{i}^{*}$ satisfies $\bar{\pi}_{i}^{z_{1}^{i}}\left(c_{i}^{*}\right)=\bar{\pi}_{i}^{z_{2}^{i}}\left(c_{i}^{*}\right)$; $p^{-i}(p) \in(0,1)$ and is determined by the condition $\left(p^{-i}(p)\right)^{z_{1}} \frac{d \bar{\pi}_{i}^{z_{i}}\left(c_{i}^{*}\right)}{d c_{i}}+\left(1-\left(p^{-i}(p)\right)^{z_{2}}\right) \frac{d \bar{\pi}_{i}^{z_{2}}\left(c_{i}^{*}\right)}{d c_{i}} \in$ $\partial V_{i}\left(\hat{c}_{i}\right)$. 
This completes our presentation of some of the environments that satisfy (9).

We now continue with the characterization of revenue maximizing mechanisms. Given (9), $V_{i}\left(\bar{c}_{i} ; p, p^{-i}(p)\right)$ can be written as

$$
V_{i}\left(\bar{c}_{i} ; p, p^{-i}(p)\right)=\underline{U}_{i}\left(c_{i}^{*}, p^{-i}\right)+\int_{c_{i}^{*}}^{\bar{c}_{i}} P_{i}(s) d s .
$$

Then substituting (10) into (8), the objective function of the seller's problem can be rewritten as

$$
\int_{C} \sum_{z \in Z} p^{z}(c) J_{z}(c) f(c) d c-\sum_{i=1}^{I}\left[\underline{U}_{i}\left(c_{i}^{*}, p^{-i}\right)+\int_{c_{i}^{*}}^{\bar{c}_{i}} P_{i}(s) d s\right] .
$$

We define the "modified virtual surplus" of allocation $z$ by

$$
\hat{J}_{z}(c)=J_{z}(c)-\sum_{i=1}^{I} 1_{c_{i} \geq c_{i}^{*}} \frac{\partial \pi_{i}^{z}(c)}{\partial c_{i}} \frac{1}{f_{i}\left(c_{i}\right)} .
$$

Then substituting (12) into (11) and recalling that $P_{i}\left(c_{i}\right)=\int_{C_{-i}} \sum_{z \in Z} p^{z}(c) \frac{\partial \pi_{i}^{z}\left(c_{i}, c_{-i}\right)}{\partial c_{i}} f_{-i}\left(c_{-i}\right) d c_{-i}$, we can rewrite the seller's objective function as

$$
\int_{C} \sum_{z \in Z} p^{z}(c) \hat{J}_{z}(c) f(c) d c-\sum_{i=1}^{I} \underline{U}_{i}\left(c_{i}^{*}, p^{-i}\right) .
$$

These observations are summarized in the following proposition

Proposition 7 If (9) is satisfied, the seller's expected revenue can be expressed as a linear function of the assignment rule,

$$
\int_{C} \sum_{z \in Z} p^{z}(c) \hat{J}_{z}(c) f(c) d c-\sum_{i=1}^{I} \underline{U}_{i}\left(c_{i}^{*}, p^{-i}\right),
$$

where $\hat{J}_{z}$ is the modified virtual surplus of allocation $z$ defined in (12).

Proposition 7 allows one to break the characterization of revenue maximizing mechanisms into two steps: first find an optimal assignment rule, $p$, and then an optimal nonparticipation assignment rule $\left\{p^{-i}\right\}_{i \in I}$.

In the first step we look for an assignment rule $p$ that solves:

$$
\begin{aligned}
& \max _{p \in \Delta(Z)} \int_{C} \sum_{z \in Z} p^{z}(c) \hat{J}_{z}(c) f(c) d c \\
& \text { s.t. } P_{i} \text { increasing. }
\end{aligned}
$$


This part of the problem has a similar structure as the classical problem in Myerson (1981), but with modified virtual surpluses, and can be solved using relatively conventional methods. Despite this, the solution will often exhibit stark differences from the solution to the classical problem. We first state the solution, and then discuss the differences.

\section{Step 1: Find the optimal $p$}

The solution is straightforward if the assignment rule that solves the relaxed program

$$
\max _{p \in \Delta(Z)} \int_{C} \sum_{z \in Z} p^{z}(c) \hat{J}_{z}(c) f(c) d c
$$

also satisfies the requirement of $P_{i}$ being increasing. This is so because the relaxed program can be solved by pointwise maximization. Following Myerson (1981) we will refer to this as the regular case. On the other hand, in the general case, pointwise optimization will lead to a mechanism that may not be feasible.

In the classical problem, a sufficient condition for the problem to be regular is that the virtual valuations are increasing. A mild condition on the distribution function $F_{i}$, $(M H R)$ guarantees that. Unfortunately, in our more general environment the problem fails to be regular even if virtual valuations (or modified virtual surpluses) are monotonic. In this case Myerson's (1981) technique of obtaining 'ironed' virtual valuations will not work. In Figueroa and Skreta (2006b) we illustrate a way to solve the general case, which does not impose additional assumptions, such as differentiability, on the mechanism. There we argue that in the general case an optimal mechanism will involve randomizations between allocations. Such lotteries are quite surprising given that buyers are risk neutral and types are single dimensional.

We now state a condition, that is very intuitive, and guarantees that pointwise optimization will lead to a feasible solution. This condition generalizes the one in Myerson (1981): with independent private values and linear utility functions our condition is satisfied whenever MHR is satisfied.

Recall that $I C$ requires the derivative of $V_{i}$, more precisely a selection from its subgradient, $P_{i}$, to be increasing in $c_{i}$. Pointwise optimization assigns probability one to the allocation with the highest virtual surplus at each vector of types. Along a region where there is no switch an allocation $z$ is selected throughout and $P_{i}\left(c_{i}\right)=\int_{C_{-i}} \frac{\partial \pi_{i}^{z}\left(c_{i}, c_{-i}\right)}{\partial c_{i}} f_{-i}\left(c_{-i}\right) d c_{-i} \equiv$ $P_{i}^{z}\left(c_{i}\right)$ is increasing by the convexity of $\pi_{i}$. Incentive compatibility can be violated though, when the seller wishes to switch, say, from allocation $z_{1}$ to $z_{2}$. At such a point $c$ we have that $\hat{J}_{z_{2}}(c) \geq \hat{J}_{z_{1}}(c)$ and $I C$ requires that $P_{i}$ does not decrease, namely $P_{i}^{z_{2}}\left(c_{i}\right) \geq P_{i}^{z_{1}}\left(c_{i}\right)$. Our condition guarantees precisely this. 
Assumption $8{ }^{23}$ Let $z_{1}, z_{2} \in Z$ be any two allocations. For a given cost realization $\left(c_{i}, c_{-i}\right)$ if $z_{1} \in \arg \max _{z \in Z} \hat{J}_{z}\left(c_{i}^{-}, c_{-i}\right)$ and $z_{2} \in \arg \max _{z \in Z} \hat{J}_{z}\left(c_{i}^{+}, c_{-i}\right)$, then $n^{24}$

$$
\frac{\partial \pi_{i}^{z_{2}}\left(c_{i}, c_{-i}\right)}{\partial c_{i}} \geq \frac{\partial \pi_{i}^{z_{1}}\left(c_{i}, c_{-i}\right)}{\partial c_{i}}
$$

Moreover, for linear environments with independent private values, there is a milder condition, for which MHR is sufficient:

Lemma 9 If the payoff functions are of the form $\pi_{i}^{z}\left(c_{i}, c_{-i}\right) \equiv A_{i}^{z}+B_{i}^{z} c_{i}$, and $\frac{F_{i}\left(c_{i}\right)}{f_{i}\left(c_{i}\right)}$ is increasing in $c_{i}$ for all $i$, then Assumption (8) is satisfied.

For general environments there is also another condition, which is more stringent but easier to verify than Assumption (8). Its statement and proof of sufficiency can be found in the appendix.

With the help of Assumption 8, it is straightforward to find an optimal assignment rule which is described in the following result.

Theorem 10 Suppose that (9) holds. If Assumption 8 is satisfied, then an optimal allocation $p$ is given by: ${ }^{25}$

$$
p^{z^{*}}(c)=\left\{\begin{array}{ll}
1 & \text { if } z^{*} \in \arg \max _{z} \hat{J}_{z}(c) \\
0 & \text { otherwise }
\end{array} .\right.
$$

We continue by characterizing revenue maximizing non-participation assignment rules.

\section{Step 2: Find optimal $p^{-i}$}

Once we have the optimal $p$ from Step 1 , it is very easy to pin down $p^{-i}$. An optimal $p^{-i}$ minimizes

$$
\underline{U}_{i}\left(c_{i}^{*}, p^{-i}\right)=E_{c_{-i}}\left[\sum_{z \in Z^{-i}}\left(p^{-i}\right)^{z}\left(c_{-i}\right) \pi_{i}^{z}\left(c_{i}^{*}, c_{-i}\right)\right],
$$

where $c_{i}^{*}$ satisfies (5) for all $p$ and $p^{-i}$. Now (5) implies that if $c_{i}^{*}$ is interior, $V_{i}$ and $\underline{U}_{i}$ must be parallel at $c_{i}^{*}$, and the seller will choose a different $p^{-i}(p)$ for different allocation rules $p$, even though the participation constraint will always bind at the same $c_{i}^{*}$. If we are at a corner, that is $c_{i}^{*}=\bar{c}_{i}$ or $c_{i}^{*}=\underline{c}_{i}, V_{i}$ and $\underline{U}_{i}$ need not be parallel at $c_{i}^{*}$, and the optimal $p^{-i}$ assigns probability one to the allocation that minimizes $E_{c_{-i}}\left[\pi_{i}^{z}\left(c_{i}^{*}, c_{-i}\right)\right]$ and $p^{-i}$ is independent of $p$.

\footnotetext{
${ }^{23}$ This condition has similar flavor to condition 5.1 in the environment of Jehiel and Moldovanu (2001b). We are grateful to Benny Moldovanu for bringing to our attention this connection.

${ }^{24}$ The notation $c_{i}^{-}$means limit from the left to $c_{i}$ and $c_{i}^{+}$means limit from the right to $c_{i}$.

${ }^{25}$ Ties are broken arbitrarily.
} 
Theorem 11 Suppose that for every $p$, all solutions of (7), satisfy (9) for some $c_{i}^{*} \in\left[\underline{c}_{i}, \bar{c}_{i}\right]$. Then, if $c_{i}^{*}=\bar{c}_{i}$ or $c_{i}^{*}=\underline{c}_{i}$, the solution of (7) $p^{-i}(p)$ is independent of $p$ and it is given $b y^{26}$

$$
\left(p^{-i}\right)^{z_{-i}}=\left\{\begin{array}{l}
1 \text { for } z_{-i} \text { that solve } \min _{z \in Z^{-i}} E_{c_{-i}}\left[\pi_{i}^{z}\left(c_{i}^{*}, c_{-i}\right)\right] \\
0 \text { otherwise }
\end{array} .\right.
$$

If $c_{i}^{*} \in\left(\underline{c}_{i}, \bar{c}_{i}\right)$, then $p^{-i}$ depends on $p$ and it satisfies

$$
p^{-i} \in \arg \min _{\rho^{-i} \in \mathcal{P}^{-i}} \underline{U}_{i}\left(c_{i}^{*}, p^{-i}\right) \text { and } \frac{\partial \underline{U}_{i}\left(c_{i}^{*}, p^{-i}\right)}{\partial c_{i}} \in \partial V_{i}\left(c_{i}^{*}\right) .{ }^{27}
$$

If Assumption 8 is not satisfied, pointwise optimization fails to lead to an incentive compatible mechanism. In Figueroa and Skreta (2006b) we show that in this case the optimal assignment rule may randomize between allocations.

The qualitative features of the solution depend on whether the conditions in (9) are satisfied for $c_{i}^{*}=\bar{c}_{i}, c_{i}^{*}=\underline{c}_{i}$, or $c_{i}^{*} \in\left(\underline{c}_{i}, \bar{c}_{i}\right)$.

When they are satisfied for $c_{i}^{*}=\bar{c}_{i}$, then it follows that $\hat{J}_{z}(c)=J_{z}(c)$ which, since $\frac{F_{i}\left(c_{i}\right)}{f_{i}\left(c_{i}\right)} \frac{\partial \pi_{i}^{z}\left(c_{i}, c_{-i}\right)}{\partial c_{i}}<0$, is always smaller than the actual surplus of allocation $z$ given by $\left.S_{z}(c)=\sum_{i=1}^{I} \pi_{i}^{z}\left(c_{i}, c_{-i}\right)\right)$. Then the seller sells less often than it is efficient.

When they are satisfied for $c_{i}^{*}=\underline{c}_{i}$ for all $i$, then

$$
\hat{J}_{z}(c)=J_{z}(c)-\sum_{i=1}^{I} \frac{\partial \pi_{i}^{z}(c)}{\partial c_{i}} \frac{1}{f_{i}\left(c_{i}\right)}
$$

which can be rewritten as

$$
\hat{J}_{z}(c)=\sum_{i=1}^{I}\left[\pi_{i}^{z}\left(c_{i}, c_{-i}\right)+\frac{F_{i}\left(c_{i}\right)-1}{f_{i}\left(c_{i}\right)} \frac{\partial \pi_{i}^{z}\left(c_{i}, c_{-i}\right)}{\partial c_{i}}\right] .
$$

In this case $\hat{J}_{z}(c)>J_{z}(c)$ because $\sum_{i=1}^{I} \frac{\partial \pi_{i}^{z}(c)}{\partial c_{i}} \frac{1}{f_{i}\left(c_{i}\right)}$ is negative. Moreover since the amount $\left(\frac{F_{i}\left(c_{i}\right)-1}{f_{i}\left(c_{i}\right)} \frac{\partial \pi_{i}^{z}\left(c_{i}, c_{-i}\right)}{\partial c_{i}}\right)$ is positive, the "modified virtual surplus" of allocation $z$, is actually larger than the actual surplus of allocation $z, S_{z}(c)$. This phenomenon will imply that in many environments a revenue maximizing mechanism sells more than it is efficient. This is in contrast with a standard intuition from monopoly theory, where the monopolist restricts supply in order to generate higher revenue.

Corollary 12 Suppose that when the seller keeps all objects, every buyer gets a payoff independent of his type. Then, at a revenue maximizing assignment rule the seller keeps all the objects less often compared to what is ex-post efficient. ${ }^{28}$

\footnotetext{
${ }^{26}$ Ties are broken arbitrarily.

${ }^{27}$ Notice that $\underline{U}_{i}$ is differentiable, since it is a linear combination of differentiable functions.

${ }^{28}$ This just one of the instances where overselling occurs.
} 
Finally, when (9) are satisfied for a $c_{i}^{*} \in\left(\underline{c}_{i}, \bar{c}_{i}\right)$, then it is possible that $\hat{J}_{z}(c)<S_{z}(c)$; or $\hat{J}_{z}(c)>S_{z}(c)$; or even $\hat{J}_{z}(c)=S_{z}(c)$, in which case a revenue maximizing auction is ex-post efficient. This is the case in Example 6.2.

\subsection{Optimal Mechanisms when $V_{i}\left(\bar{c}_{i} ; p, p^{-i}(p)\right)$ is nonlinear in $p$}

When $V_{i}\left(\bar{c}_{i} ; p, p^{-i}(p)\right)$ depends non-linearly in the assignment rule $p$, the seller's objective function becomes non-linear ${ }^{29}$ and the solution cannot be obtained via pointwise maximization. Fortunately, the problem has enough structure to allow the use of variational methods. In particular, if the functions $\pi_{i}^{z}\left(\cdot, c_{-i}\right)$ are smooth enough, then $c_{i}^{*}\left(p, p^{-i}(p)\right)$ is a differentiable function of $p$, thus guaranteeing that the objective function is differentiable, and hence continuous. It is not hard to show that the feasible set is sequentially compact. A continuous function over a sequentially compact set has a maximum. The solution will depend on the particular shapes of $\pi_{i}^{z}$ and of the distributions $F_{i}$. This environment is more complicated than the ones considered by Jullien (2000), not only because there are multiple agents, but more importantly because the seller can choose the outside options. However in Figueroa and Skreta (2005) we show that the problem often, (but not always), reduces to one with essentially exogenous non-participation assignment rules. Unfortunately, the difficulties arising from having a non-linear objective function are present even when the non-participation assignment rule is exogenous.

\footnotetext{
${ }^{29}$ Suppose there is one buyer and three possible allocations $z_{1}, z_{2}, z_{3}$. The payoffs of the allocations are $\pi^{z_{1}}(c)=10-10 c, \pi^{z_{2}}(c)=0$ and $\pi^{z_{3}}(c)=-5 c$, where $c \in[\underline{c}, \bar{c}]$. Suppose also that $\left(p^{-1}\right)^{z_{3}}=1$, so the non-participation assignment rule assigns probability one to allocation $z_{3}$. An assignment rule $p(c)=$ $\left(p^{z_{1}}(c), p^{z_{2}}(c), p^{z_{3}}(c)\right)$ induces a surplus
}

$$
V(c)=V\left(\bar{c} ; p, p^{-1}\right)-\int_{c}^{\bar{c}} P(s) d s
$$

which, in the points where it is differentiable satisfies $\frac{d V(c)}{d c}=P(c)=-10 p^{z_{1}}(c)-5 p^{z_{3}}(c)$. The type where the participation constraint binds depends on how $P(c)$, which is slope of the payoff from participating in the mechanism, compares to the slope of the payoff from non-participating, which is given by -5 . It is clear that the critical type $c^{*}$ depends non-linearly on $p$, and it is given by

$$
c^{*}\left(p, p^{-1}\right)= \begin{cases}\underline{c} & \text { if }-5 \leq-10 p^{z_{1}}(0)-5 p^{z_{3}}(0) \\ \bar{c} & \text { if }-5 \geq-10 p^{z_{1}}(1)-5 p^{z_{3}}(1) \\ c^{*} & \sim\end{cases}
$$

where $c^{*}$ satisfies that $-10 p^{z_{1}}\left(c^{*^{-}}\right)-5 p^{z_{3}}\left(c^{*^{-}}\right) \leq-5 \leq-10 p^{z_{1}}\left(c^{*^{+}}\right)-5 p^{z_{3}}\left(c^{*^{+}}\right)$. Since

$$
V\left(\bar{c}, p, p^{-1}\right)=-5 c^{*}\left(p, p^{-1}\right)+\int_{c^{*}\left(p, p^{-1}\right)}^{\bar{c}}\left[-10 p^{z_{1}}(c)-5 p^{z_{3}}(c)\right] d c
$$

we have that the objective function is non-linear in the assignment rule $p$. 


\section{Illustration of the Solution}

The purpose of this section is to illustrate the solution in simple but economically insightful examples.

\subsection{The Role of Steep Outside Options}

Consider 2 firms fighting for a single slot to advertise their products. There are three feasible allocations. The seller keeps the slot, $z_{0}$; buyer 1 gets the slot, $z_{1}$ or buyer 2 gets the slot, $z_{2}$.

The value of airing a spot depends on the actual cost parameter $c_{i}$ of firm $i$, which is private information and is uniformly and independently distributed in $[0,1]$ for both firms. The value of not airing a spot depends on the allocation implemented: a firm suffers an externality, (which depends on its cost parameter $c_{i}$ ) if its competitor gets the spot, while it gets a payoff of 0 in case nobody gets it. Let $\pi_{i}^{z_{j}}\left(c_{i}\right)$ denote the payoff of firm $i$ if allocation $z_{j}$ is implemented and its type is $c_{i}$. The payoffs that accrue to each firm from each of these alternatives are

$$
\begin{array}{ll}
\pi_{1}^{z_{0}}\left(c_{1}\right)=0 & \pi_{2}^{z_{0}}\left(c_{2}\right)=0 \\
\pi_{1}^{z_{1}}\left(c_{1}\right)=1-c_{1} & \pi_{2}^{z_{1}}\left(c_{2}\right)=-2 c_{2} \quad . \\
\pi_{1}^{z_{2}}\left(c_{1}\right)=-2 c_{1} & \pi_{2}^{z_{2}}\left(c_{2}\right)=1-c_{2}
\end{array} .
$$

An assignment rule here is $p(c)=\left(p^{z_{0}}(c), p^{z_{1}}(c), p^{z_{2}}(c)\right)$, where $c=\left(c_{1}, c_{2}\right)$. The seller's problem can be written as:

$$
\begin{gathered}
\max _{p} \int_{[0,1]} \int_{[0,1]}\left[p^{z_{0}}(c) J_{z_{0}}(c)+p^{z_{1}}(c) J_{z_{1}}(c)+p^{z_{2}}(c) J_{z_{2}}(c)\right] d c_{1} d c_{2}-V_{1}\left(1, p, p^{-1}(p)\right)-V_{2}\left(1, p, p^{-2}(p)\right) \\
\text { s.t. } \quad P_{1}\left(c_{1}\right) \equiv-\int\left[p^{z_{1}}(c)+2 p^{z_{2}}(c)\right] d c_{2} \text { is increasing } \\
P_{2}\left(c_{2}\right) \equiv-\int\left[2 p^{z_{1}}(c)+p^{z_{2}}(c)\right] d c_{1} \text { is increasing } \\
0 \leq p^{z_{i}}(c) \leq 1, i=0,1,2 \text { and } \Sigma_{i \in\{0,1,2\}} p^{z_{i}}(c)=1
\end{gathered}
$$

where

$$
\begin{aligned}
& J_{z_{0}}(c)=0 \\
& J_{z_{1}}(c)=1-2 c_{1}-4 c_{2} \\
& J_{z_{2}}(c)=1-2 c_{2}-4 c_{1} .
\end{aligned}
$$

The optimal assignment rule crucially depends on the allocations that prevail if a buyer refuses to participate in the mechanism, since these determine $V_{i}\left(1, p, p^{-i}(p)\right), i=1,2$.

Scenario 1: Flat Outside Options 
In this case if a buyer does not participate the seller must keep the slot. Then

$$
p^{-1}=p^{-2}=\left(p^{z_{0}}(c), p^{z_{1}}(c), p^{z_{2}}(c)\right)=(1,0,0) .
$$

Given this non-participation assignment rule, the payoff to buyer $i$ from not participation is $\pi_{i}^{z_{0}}\left(c_{i}\right)=0$ which is independent of $i^{\prime} s$ type. The participation constraint binds at the "worst" type $\bar{c}_{1}=\bar{c}_{2}=1$, since at an incentive compatible assignment rule $V_{i}$ is decreasing in $c_{i}$. See Figure 3. INSERT FIgURE 3 HeRE. This implies immediately that

$$
V_{1}\left(1, p, p^{-1}(p)\right)=V_{2}\left(1, p, p^{-2}(p)\right)=0 .
$$

Then, pointwise maximizing gives us

$$
p(c)=\left\{\begin{array}{ll}
(0,1,0) & \text { if } c_{2} \leq c_{1} \text { and } 1 \geq 2 c_{1}+4 c_{2} \\
(0,0,1) & \text { if } c_{1} \leq c_{2} \text { and } 1 \geq 2 c_{2}+4 c_{1} \\
(1,0,0) & \text { if } 2 c_{1}+4 c_{2}>1 \text { and } 2 c_{2}+4 c_{1}>1
\end{array} .\right.
$$

which is feasible, and hence optimal. See Figure 4. InSERT Figure 4 Here. Feasibility follows from Lemma 9, since we have linear payoffs and a distribution that satisfies $M H R$.

\section{Scenario 2: Steep Outside Options}

In this case, if a buyer fails to participate the seller may give the slot to its competitor, the other buyer, that is

$$
p^{-1}=\left(p^{z_{0}}(c), p^{z_{1}}(c), p^{z_{2}}(c)\right)=(0,0,1) \text { and } p^{-2}=\left(p^{z_{0}}(c), p^{z_{1}}(c), p^{z_{2}}(c)\right)=(0,1,0) .
$$

It is not hard to see that this is the optimal way for the seller to threaten buyers, since giving the slot to the competitor has the lowest payoff for buyer $i$. We now justify in detail why this is the case. Observe that allocation $z_{j}$ gives the lowest payoff to $i$ when his cost is the smallest possible, that is

$$
\pi_{i}^{z_{j}}(0) \leq \pi_{i}^{z}(0) \text { for all } z \in\left\{z_{0}, z_{1}, z_{2}\right\} .
$$

Allocation $z_{j}$ also gives the steepest payoff to buyer $i$, for $i, j=1,2$ since we have that

$$
\frac{d \pi_{i}^{z_{j}}\left(c_{i}\right)}{d c_{i}} \leq \frac{d \pi_{i}^{z}}{d c_{i}} \text { for all } z \in\left\{z_{0}, z_{1}, z_{2}\right\}
$$

From (16) and (17) it follows that the participation constraint will always bind at $c_{i}^{*}=\underline{c}_{i}=0$.

See Figure 5. InSERT Figure 5 HERE. 
Then for $i, j=1,2$ we have

$$
\begin{aligned}
V_{i}\left(1, p, p^{-i}(p)\right) & =\pi_{i}^{z_{j}}\left(c_{i}^{*}\right)+\int_{c_{i}^{*}}^{1} P_{i}\left(c_{i}\right) d c_{i} \\
& =\pi_{i}^{z_{j}}(0)+\int_{0}^{1} P_{i}\left(c_{i}\right) d c_{i} \\
& =0+\int_{0}^{1} \int_{0}^{1}\left[-p^{z_{i}}(c)-2 p^{z_{j}}(c)\right] d c .
\end{aligned}
$$

Substituting these expressions in the objective function, the seller's problem becomes

$$
\begin{array}{ll}
\max _{p} & \int_{[0,1][0,1]}\left[p^{z_{1}}(c)\left[4-2 c_{1}-4 c_{2}\right]+p^{z_{2}}(c)\left[4-2 c_{2}-4 c_{1}\right]\right] d c_{1} d c_{2} \\
\text { s.t. } & P_{1}\left(c_{1}\right) \equiv-\int\left[p^{z_{1}}(c)+2 p^{z_{2}}(c)\right] d c_{2} \text { is increasing } \\
& P_{2}\left(c_{2}\right) \equiv-\int\left[2 p^{z_{1}}(c)+p^{z_{2}}(c)\right] d c_{1} \text { is increasing } \\
& 0 \leq p^{z_{i}}(c) \leq 1, i=0,1,2 \text { and } \Sigma_{i \in\{0,1,2\}} p^{z_{i}}(c)=1 .
\end{array}
$$

So, comparing problems (15) and (19), we see that after incorporating the terms $V_{1}\left(1, p, p^{-1}(p)\right)$ and $V_{2}\left(1, p, p^{-2}(p)\right)$ we get "modified virtual surpluses", which are given by

$$
\begin{aligned}
& \hat{J}_{z_{0}}(c)=0 \\
& \hat{J}_{z_{1}}(c)=4-2 c_{1}-4 c_{2} \\
& \hat{J}_{z_{2}}(c)=4-2 c_{2}-4 c_{1} .
\end{aligned}
$$

The assignment rule corresponding to pointwise maximization is given by

$$
p(c)=\left\{\begin{array}{ll}
(0,1,0) & \text { if } c_{2} \leq c_{1} \text { and } 4 \geq 2 c_{1}+4 c_{2} \\
(0,0,1) & \text { if } c_{1} \leq c_{2} \text { and } 4 \geq 2 c_{2}+4 c_{1} \\
(1,0,0) & \text { if } 2 c_{1}+4 c_{2}>4 \text { and } 2 c_{2}+4 c_{1}>4
\end{array} .\right.
$$

This assignment rule is feasible by Lemma 9 (and therefore optimal). See Figure 6. INSERT FIGURE 6 HERE.

As discussed earlier, when participation constraints bind at the smallest cost "overselling" occurs, compared to what is efficient. In this example, the "ex-post efficient allocation" is given by

$$
p^{e}(c)= \begin{cases}(0,1,0) & \text { if } c_{2} \leq c_{1} \text { and } c_{1}+2 c_{2} \leq 1 \\ (0,0,1) & \text { if } c_{1} \leq c_{2} \text { and } 2 c_{1}+c_{2} \leq 1 \\ (1,0,0) & \text { otherwise }\end{cases}
$$

See Figure 7. InSERT Figure 7 HeRE. 
Comparing $p(c)$ and $p^{e}(c)$, depicted in Figures 6 and 7 respectively, we see that at the revenue maximizing assignment rule buyers 1 and 2 obtain the slot for cost realizations where efficiency dictates that the seller should keep it. At first sight this is in contrast with the intuition that a monopolist creates inefficiencies by restricting output, since in this case he is selling more than what it is socially desirable. In fact, there is no contradiction, what is being sold "too little" here is the (socially desirable) service of not assigning the spot.

This example illustrates that the optimal assignment rule critically depends on the outside options that each buyer faces. When the seller can only keep the object if a buyer fails to participate, the optimal assignment rule assigns the slot less often than it is efficient. In contrast, in the case where if a buyer fails to participate, the seller gives the slot to the other firm, the revenue maximizing assignment rule allocates the spot more often then it is efficient. The reason why the solution in the two scenaria under consideration differs, is that in the second one when firm $i$ fails to participate its payoff depends on its cost, $\left.\left(\pi_{i}^{z_{j}}\left(c_{i}\right)=-2 c_{i}\right)\right)$. If $\pi_{i}^{z_{j}}\left(c_{i}\right)=-2$, then the revenue maximizing assignment rule would be the same in Scenario 1 and in Scenario 2; (in this case only payments would differ). Summarizing, outside options affect the optimal assignment rule only if the payoffs from non-participation are type-dependent.

\subsection{An Example with Coexistence of Steep \& Flat Outside Options ${ }^{30}$}

A seller has an invention which is of potential interest to firm A. The firm has a cost parameter $c$ distributed uniformly in $[0,1]$. In case firm A gets the exclusive rights, its valuation is given by $\pi^{z_{A}}(c)=5-5 c$. In case that there is no sale, the seller can either keep the invention or open source it. A very efficient firm is not afraid of competition and prefers open sourcing to no sale at all, whereas a more inefficient firm prefers the opposite. ${ }^{31}$ In particular, in the case of no sale firm A gets $\pi^{z_{1}}(c)=0$, and in the case of open sourcing it gets $\pi^{z_{2}}(c)=1-10 c$. So if firm $\mathrm{A}$ is very efficient with $c \leq \frac{1}{10}$, the option of no one obtaining the invention is worse than open source. The opposite is true when $c \geq \frac{1}{10}$. See Figure 8. INSERT FigURE 8 HERE. An assignment rule here is $p(c)=\left(p^{z_{A}}(c), p^{z_{1}}(c), p^{z_{2}}(c)\right)$. In case firm $A$ does not participate in the sale, the seller is indifferent between keeping the invention and making it open source. In fact, since there is nothing else the seller can do in that case, any randomization between these options is optimal from her perspective and hence credible.

The seller solves:

$$
\begin{array}{cl}
\max _{p} & \int_{0}^{1}\left[p^{z_{A}}(c)[5-10 c]+p^{z_{2}}(c)[1-20 c]\right] d c-V\left(1, p, p^{-i}(p)\right) \\
\text { s.t. } & -\left[5 p^{z_{A}}(c)+10 p^{z_{2}}(c)\right] \text { is increasing } \\
& 0 \leq p^{z}(c) \leq 1 \text { for all } z \in\left\{z^{A}, z^{1}, z^{2}\right\} \text { and } \Sigma_{z \in\left\{z^{A}, z^{1}, z^{2}\right\}} p^{z}(c)=1
\end{array}
$$

\footnotetext{
${ }^{30}$ This is essentially the example described in the introduction.

${ }^{31}$ This is different from the previous example, where the allocation that hurts buyers the most is always the same.
} 
This example belongs to the class of problems which satisfy (9) for $c_{i}^{*} \in\left(\underline{c}_{i}, \bar{c}_{i}\right)$ discussed on Proposition 6. As already discussed, in this case an optimal non-participation rule $p^{-A}$ depends on the assignment rule $p$ that the seller wants to implement. We therefore start by specifying the optimal $p^{-A}$ as a function of $p$ and then solve for an optimal $p$.

1. Finding optimal $p^{-A}(p)$

With a slight abuse of notation, let $p^{-A}$ denote the probability that allocation $z_{2}$ is chosen if $\mathrm{A}$ fails to participate, and let $\left(1-p^{-A}\right)$ the probability that allocation $z_{1}$ will be chosen. Associated with this non-participation assignment rule is the payoff that will accrue to $\mathrm{A}$ if it fails to participate

$$
\begin{aligned}
\underline{U}_{A}\left(c, p^{-A}\right) & =\left(1-p^{-A}\right) \cdot 0+p^{-A}(1-10 c) \\
& =p^{-A}-10 p^{-A} c .
\end{aligned}
$$

We know that the optimal non-participation assignment rule must minimize

$$
V\left(1, p, p^{-A}(p)\right)=\underline{U}_{A}\left(c^{*}\left(p, \rho^{-A}\right), p^{-A}\right)+\int_{c^{*}\left(p, \rho^{-A}\right)}^{1} \frac{d V(c)}{d c} d c,
$$

which using (21) can be rewritten as

$$
V\left(1, p, p^{-A}(p)\right)=\rho^{-A}-10 \rho^{-A} c^{*}\left(p, \rho^{-A}\right)+\int_{c^{*}\left(p, \rho^{-A}\right)}^{1} \frac{d V(c)}{d c} d c .
$$

Now at a solution ${ }^{32} p^{-A}(p)$ the total derivative of $V\left(1, p, p^{-i}(p)\right)$ with respect to $\rho^{-A}$ is equal to the partial, and it is given by

$$
\left.\frac{d V\left(1, p, p^{-A}(p)\right)}{d \rho^{-A}}\right|_{\rho^{-A}=p^{-A}(p)}=1-10 c^{*}\left(p, p^{-A}(p)\right) .
$$

Moreover at an interior minimum it must be the case that

$$
\left.\frac{d V\left(1, p, p^{-A}(p)\right)}{d \rho^{-A}}\right|_{\rho^{-A}=p^{-A}(p)}=1-10 c^{*}\left(p, p^{-A}(p)\right)=0,
$$

which implies that

$$
c^{*}\left(p, p^{-A}(p)\right)=\frac{1}{10} \text { for all } p \text { and } p^{-A} .
$$

We have therefore verified that this example satisfies (9) for $c_{i}^{*}=\frac{1}{10}$, and hence the critical type is independent of $p$ and $p^{-A}$.

\footnotetext{
${ }^{32}$ This property has a flavor of envelope condition. We state it formally in Lemma A in the Appendix.
} 
We proceed to find an optimal $p^{-A}$ as a function of an assignment rule $p$. The slope of the payoff from non-participation is

$$
\frac{\partial \underline{U}_{A}\left(c, p^{-A}\right)}{\partial c}=-10 p^{-A}
$$

At an optimal $p^{-A}$ this has to be equal to the slope of the participation payoff $V$ at $c^{*}\left(p, p^{-A}(p)\right)$ which in our case it is $\frac{1}{10}$. In other words

$$
\left.\frac{d V(c)}{d c}\right|_{c^{*}=\frac{1}{10}}=-10 p^{-A}
$$

now given an assignment rule $p(c)=\left(p^{z_{A}}(c), p^{z_{1}}(c), p^{z_{2}}(c)\right), V(c)$ is given by

$$
V(c)=p^{z_{A}}(c)(5-5 c)+p^{z_{1}}(c) \cdot 0+p^{z_{2}}(c)(1-10 c),
$$

and its slope is given by

$$
\frac{d V(c)}{d c}=-5 p^{z_{A}}(c)-10 p^{z_{2}}(c)
$$

With the help of (25), (24) can be rewritten as

$$
-10 p^{-A}=-5 p^{z_{A}}\left(\frac{1}{10}\right)-10 p^{z_{2}}\left(\frac{1}{10}\right),
$$

which reduces to

$$
p^{-A}(p)=\frac{1}{2} p^{A}\left(\frac{1}{10}\right)+p^{z_{2}}\left(\frac{1}{10}\right) .
$$

Equation (26) gives us an optimal $p^{-A}$ as a function of the assignment rule $p .^{33}$

\section{Finding optimal $p$}

With the help of (23), $V\left(1, p, p^{-i}(p)\right)$ given by (22), can be rewritten as $V\left(1, p, p^{-i}(p)\right)=$ $-\int_{\frac{1}{10}}^{1}\left[5 p^{z_{A}}(c)+10 p^{z_{2}}(c)\right] d c$, and substituting this into (20), the seller's problem can be rewritten as

\footnotetext{
${ }^{33}$ This example highlights the interdependence of optimal non-participation assignment rules with the assignment rules. This feature is novel and does not appear in the earlier work, (see for instance JMS (1996)), where optimal threats are independent from the way the seller wants to allocate the goods. Here equation (26) tells us that for different assignment rules, the optimal, from the seller's point of view, nonparticipation assignment rule, is different. For example, for
}

$$
\left(\tilde{p}^{z_{A}}(c), \tilde{p}^{z_{1}}(c), \tilde{p}^{z_{2}}(c)\right)= \begin{cases}(1,0,0) & \text { if } c \in\left[0, \frac{1}{2}\right] \\ (0,1,0) & \text { if } c \in\left[\frac{1}{2}, 1\right]\end{cases}
$$

the optimal non-participation assignment rule is $p^{-A}(\tilde{p})=\frac{1}{2}$. If the assignment rule is instead

$$
\left(\hat{p}^{z_{A}}(c), \hat{p}^{z_{1}}(c), \hat{p}^{z_{2}}(c)\right)= \begin{cases}\left(\frac{1}{2}, 0, \frac{1}{2}\right) & \text { if } c \in\left[0, \frac{1}{2}\right] \\ (0,1,0) & \text { if } c \in\left[\frac{1}{2}, 1\right]\end{cases}
$$

the optimal non-participation assignment rule is $p^{-A}(\hat{p})=\frac{3}{4}$. 


$$
\begin{aligned}
\max _{p} & \left.\int_{0}^{\frac{1}{10}}\left[p^{z_{A}}(c)[5-10 c]+p^{z_{2}}(c)[1-20 c]\right] d c+\int_{\frac{1}{10}}^{1} p^{z_{A}}(c)[10-10 c]+p^{z_{2}}(c)[11-20 c]\right] d c \\
\text { s.t. } & -\left[5 p^{z_{A}}(c)+10 p^{z_{2}}(c)\right] \text { is increasing } \\
& 0 \leq p^{z}(c) \leq 1 \text { for all } z \in\left\{z^{A}, z^{1}, z^{2}\right\} \text { and } \Sigma_{z \in\left\{z^{A}, z^{1}, z^{2}\right\}} p^{z}(c)=1
\end{aligned}
$$

Pointwise maximization gives us that $p^{z_{A}}(c)=1$ for all $c$, and the optimal assignment rule is:

$$
p(c)=\left(p^{z_{A}}(c)=1, p^{z_{1}}(c)=0, p^{z_{2}}(c)=0\right)
$$

which is feasible. By substituting (27) into (26), we get that the optimal non-participation assignment rule is given by

$$
\begin{aligned}
& p^{-A}(p)=\frac{1}{2} \text { or more precisely } \\
& p^{-A}(p)=\left(p^{z_{A}}(c), p^{z_{1}}(c), p^{z_{2}}(c)\right)=\left(0, \frac{1}{2}, \frac{1}{2}\right) .
\end{aligned}
$$

In this example the revenue maximizing assignment rule is ex-post efficient. To see this, notice that $\pi^{z_{A}}(c) \geq \pi^{z_{1}}(c)$ and $\pi^{z_{A}}(c) \geq \pi^{z_{2}}(c)$ for all $c \in[0,1]$, so it's always efficient to sell the invention to the firm. This efficiency property is rather surprising given the presence of private information that is statistically independent. The seller's expected revenue is 4.5. See Figure 9. INSERT FIGURE 9 HERE.

It is interesting to compare this solution to the one when open sourcing (allocation $z_{2}$ ) is not an available option. In this case the optimal assignment rule is

$$
p(c)=\left(p^{z_{A}}(c), p^{z_{1}}(c)\right)=\left\{\begin{array}{ll}
(1,0) & \text { if } c \in\left[0, \frac{1}{2}\right] \\
(0,1) & \text { if } c \in\left[\frac{1}{2}, 1\right]
\end{array},\right.
$$

and trivially the non-participation assignment rule is $p^{-A}(p)=(0,1)$. Then the seller's expected revenue is 1.25. This assignment rule is inefficient, since half of the time Firm A does not obtain the invention, whereas it is always efficient that it does. Comparing to the previous case, we see that the option of open sourcing increases both the seller's revenue, (it more than triples), and efficiency. This is despite the fact that open sourcing is never implemented. For an illustration of the solution see Figure 10. INSERT FIGURE 10 HERE.

This example highlights an important new insight. When the payoff from non-participation depends on a buyer's type, even allocations that are never implemented can crucially affect the revenue maximizing assignment of the objects. Compare Figures 9 and 10. The introduction of the option of open sourcing increased the revenue of the seller, and made the revenue maximizing assignment rule ex-post efficient, even though it is never implemented. This example also shows that optimal non-participation assignment rules can be random. 


\section{Concluding Remarks}

In this paper we study the optimal allocation mechanism for $N$ objects, to $I$ potential buyers (firms). Payoff functions allow for goods to be complements, substitutes and type dependent externalities among buyers. Private information is single-dimensional, which makes the problem tractable despite its generality. Our analysis shows that key intuitions from earlier work on optimal auctions fail to generalize. It is widely believed that efficiency and revenue maximization are conflicting objectives. However, here we show that a revenue maximizing mechanism sometimes will allocate the objects in an ex-post efficient way, and sometimes is will sell "too often". The broad message is that type-dependent non-participation payoffs change the nature of the distortions that arise from the presence of asymmetric information. This highlights the designer's role of creating the "appropriate" outside options, since they allow her to increase her revenue and the overall efficiency of the mechanism. This paper also encompasses a large number of important allocation problems as a special case. Potential applications range from allocation of airport take-off and landing slots, to allocation of positions in teams.

\section{APPEndix}

\section{Proof of Lemma $\mathbf{1}^{34}$}

By the convexity of $\pi_{i}^{z}\left(\cdot, c_{-i}\right)$ we have that $V_{i}$ is a maximum of convex functions, so it is convex, and therefore differentiable a.e. It is also easy to check that the following are equivalent:

(a) $(p, x)$ is incentive compatible

(b) $P_{i}\left(c_{i}\right) \in \partial V_{i}\left(c_{i}\right)$

(c) $U\left(c_{i}, c_{i} ;(p, x)\right)=V_{i}\left(c_{i}\right)$

We now use these equivalent statements to prove necessity and sufficiency in our Lemma.

$(\Longrightarrow)$ Here we use the fact that Incentive compatibility implies (b). A result in Krishna and Maenner (2001) then implies (3). By the convexity of $V_{i}$, we know that $\partial V_{i}$ is monotone, so:

$$
\left(P_{i}\left(c_{i}\right)-P_{i}\left(c_{i}^{\prime}\right)\right)\left(c_{i}-c_{i}^{\prime}\right) \geq 0 .
$$

This immediately implies (2).

$(\Longleftarrow)$ To prove that $(2)$ implies incentive compatibility it's enough to show that $P_{i}\left(c_{i}\right) \in$

\footnotetext{
${ }^{34}$ This proof is relatively standard, see for instance, Jehiel, Moldovanu and Stacchetti (1999) and is included for completeness.
} 
$\partial V_{i}\left(c_{i}\right)$. By $(2)$ and $(3)$,

$$
\begin{aligned}
V_{i}\left(c_{i}^{\prime}\right)-V_{i}\left(c_{i}\right) & =\int_{c_{i}}^{c_{i}^{\prime}} P_{i}(s) d s \\
& \geq P_{i}\left(c_{i}\right)\left(c_{i}^{\prime}-c_{i}\right)
\end{aligned}
$$

which shows $P_{i}\left(c_{i}\right) \in \partial V_{i}\left(c_{i}\right)$.

\section{Expected Payment at an Incentive Compatible Mechanism ${ }^{35}$}

Integrating the second condition in (1) over $C_{-i}$ we get that

$$
\int_{C} x_{i}(c) f(c) d c=\int_{C} \sum_{z \in Z} p^{z}(c) \pi_{i}^{z}(c) f(c) d c-\int_{C_{i}} V_{i}\left(c_{i}\right) d c_{i},
$$

and changing the order of integration we get:

$$
\begin{aligned}
\int_{C_{i}} V_{i}\left(c_{i}\right) d c_{i} & =\int_{C_{i}}\left[V_{i}\left(\overline{c_{i}}\right)-\int_{c_{i}}^{\overline{c_{i}}} P_{i}\left(s_{i}\right) d s_{i}\right] f_{i}\left(c_{i}\right) d c_{i} \\
& =V_{i}\left(\overline{c_{i}}\right)-\int_{C_{i}} P_{i}\left(s_{i}\right) \int_{c_{i}}^{s_{i}} f_{i}\left(c_{i}\right) d c_{i} d s_{i} \\
& =V_{i}\left(\overline{c_{i}}\right)-\int_{C_{i}} P_{i}\left(c_{i}\right) F_{i}\left(c_{i}\right) d c_{i} \\
& =V_{i}\left(\overline{c_{i}}\right)-\int_{C_{i}} \int_{C_{-i}} \sum_{z \in Z} p^{z}\left(c_{i}, c_{-i}\right) \frac{\partial \pi_{i}^{z}\left(c_{i}, c_{-i}\right)}{\partial c_{i}} f_{-i}\left(c_{-i}\right) d c_{-i} F_{i}\left(c_{i}\right) d c_{i} \\
& =V_{i}\left(\overline{c_{i}}\right)-\int_{C} \sum_{z \in Z} p^{z}\left(c_{i}, c_{-i}\right) \frac{\partial \pi_{i}^{z}\left(c_{i}, c_{-i}\right)}{\partial c_{i}} \frac{F_{i}\left(c_{i}\right)}{f_{i}\left(c_{i}\right)} f(c) d c
\end{aligned}
$$

From the last expression the result follows.

\section{Proof of Proposition 4}

First we prove that, if the participation constraint is satisfied at $c_{i}=\underline{c}_{i}$ then it is satisfied for all $c_{i} \in C_{i}$. This follows from three observations.

(i) $P_{i}\left(c_{i}\right) \in \partial V_{i}\left(c_{i}\right)$,

\footnotetext{
${ }^{35}$ This proof is very standard and is included for completeness.
} 
(ii)

$$
\begin{aligned}
P_{i}\left(c_{i}\right) & =\int_{C_{-i}} \sum_{z \in Z} p^{z}(c) \frac{\partial \pi_{i}^{z}\left(c_{i}, c_{-i}\right)}{\partial c_{i}} f_{-i}\left(c_{-i}\right) d c_{-i} \\
& \geq \int_{C_{-i}} \sum_{z \in Z} p^{z}(c) \frac{\partial \pi_{i}^{z_{i}^{*}}\left(c_{i}, c_{-i}\right)}{\partial c_{i}} f_{-i}\left(c_{-i}\right) d c_{-i} \\
& =\frac{\partial \bar{\pi}_{i}^{z_{i}^{*}}\left(c_{i}\right)}{\partial c_{i}}
\end{aligned}
$$

and (iii) $V_{i}\left(\underline{c}_{i}\right) \geq \bar{\pi}_{i}^{z_{i}^{*}}\left(\underline{c}_{i}\right)$.

Observations (i) and (ii) imply that the derivative of $V_{i}$ is always greater than the derivative of $\bar{\pi}_{i}^{z_{i}^{*}}$. These two, together with (iii) imply that $V\left(c_{i}\right) \geq \bar{\pi}_{i}^{z_{i}^{*}}\left(c_{i}\right)$ for all $c_{i} \in C_{i}$.

\section{Proof of Proposition 6}

To prove this proposition, we first prove the following lemma:

Lemma A.

$$
\left.\frac{d V_{i}\left(\bar{c}_{i} ; p, \rho^{-i}\right)}{d\left(\rho^{-i}\right)^{z}}\right|_{\rho^{-i}=p^{-i}(p)}=\left.\frac{\partial V_{i}\left(\bar{c}_{i} ; p, \rho^{-i}\right)}{\partial\left(\rho^{-i}\right)^{z}}\right|_{\rho^{-i}=p^{-i}(p)}=\bar{\pi}_{i}^{z}\left(c_{i}^{*}\left(p, p^{-i}(p)\right)\right) \text {, for all } z \in Z^{-i} .
$$

Proof. We suppose for simplicity that the derivative $\frac{\partial c_{i}^{*}\left(p, \rho^{-i}\right)}{\partial \rho^{-i}}$ is well defined, (otherwise we can do all the analysis with subgradients).

Then, differentiating $V_{i}\left(\bar{c}_{i} ; p, \rho^{-i}\right)$ with respect to $\left(\rho^{-i}\right)^{z}$ we obtain that

$$
\frac{\partial V_{i}\left(\bar{c}_{i} ; p, \rho^{-i}\right)}{\partial\left(\rho^{-i}\right)^{z}}=\frac{\partial \underline{U}_{i}\left(c_{i}^{*}\left(p, \rho^{-i}\right), \rho^{-i}\right)}{\partial\left(\rho^{-i}\right)^{z}}+\left[\frac{\partial \underline{U}_{i}\left(c_{i}^{*}\left(p, \rho^{-i}\right), \rho^{-i}\right)}{\partial c_{i}}-P_{i}\left(c_{i}^{*}\left(p, \rho^{-i}\right)\right)\right] \frac{\partial c_{i}^{*}\left(p, \rho^{-i}\right)}{\partial\left(\rho^{-i}\right)^{z}}
$$

Given an assignment rule $p$ and a non-participation assignment rule $\rho^{-i}$, we know that at an optimal mechanism $c_{i}^{*}\left(p, \rho^{-i}\right)$ satisfies $c_{i}^{*}\left(p, \rho^{-i}\right) \in \arg \min _{c_{i}}\left[-\int_{c_{i}}^{\bar{c}_{i}} P_{i}(s) d s-\underline{U}_{i}\left(c_{i}, \rho^{-i}\right)\right]$. Depending on whether $c_{i}^{*}\left(p, \rho^{-i}\right) \in\left(\underline{c_{i}}, \bar{c}_{i}\right)$, or $c_{i}^{*}\left(p, \rho^{-i}\right)=\underline{c_{i}}$ or $c_{i}^{*}\left(p, \rho^{-i}\right)=\bar{c}_{i}$, there are three cases to consider.

Case 1: $c_{i}^{*}\left(p, \rho^{-i}\right) \in\left(\underline{c}_{i}, \bar{c}_{i}\right)$

Since $c_{i}^{*}\left(p, \rho^{-i}\right) \in \arg \min _{c_{i}}\left[-\int_{c_{i}}^{\bar{c}_{i}} P_{i}(s) d s-\underline{U}_{i}\left(c_{i}, \rho^{-i}\right)\right]$, an interior solution (which is precisely the case under investigation), must satisfy

$$
\left.\frac{d V_{i}\left(c_{i}\right)}{d c_{i}}\right|_{c_{i}=c_{i}^{*}\left(p, \rho^{-i}\right)}=\left.\frac{\partial \underline{U}_{i}\left(c_{i}\left(p, \rho^{-i}\right), \rho^{-i}\right)}{\partial c_{i}}\right|_{c_{i}=c_{i}^{*}\left(p, \rho^{-i}\right)} .
$$


Then recall that $V_{i}\left(c_{i}\right)=V_{i}\left(\bar{c}_{i} ; p, \rho^{-i}\right)-\int_{c_{i}}^{\overline{c_{i}}} P_{i}(s) d s$, which implies that

$$
\left.\frac{d V_{i}\left(c_{i}\right)}{d c_{i}}\right|_{c_{i}=c_{i}^{*}\left(p, \rho^{-i}\right)}=-P_{i}\left(c_{i}^{*}\left(p, \rho^{-i}\right)\right)
$$

Then, substituting (30) and (31) into (29), we obtain that

$$
\left.\frac{\partial V_{i}\left(\bar{c}_{i} ; p, \rho^{-i}\right)}{\partial\left(\rho^{-i}\right)^{z}}\right|_{\rho^{-i}=p^{-i}(p)}=\frac{\partial \underline{U}_{i}\left(c_{i}^{*}\left(p, p^{-i}(p)\right), p^{-i}(p)\right)}{\partial\left(\rho^{-i}\right)^{z}}=\bar{\pi}_{i}^{z}\left(c_{i}^{*}\left(p, p^{-i}(p)\right)\right) \text {, for all } z \in Z^{-i},
$$

which is what we wanted to show.

Case 2: $c_{i}^{*}\left(p, \rho^{-i}\right)=\underline{c_{i}}$

If $p$ and $\rho^{-i}$ such that $c_{i}^{*}\left(p, \rho^{-i}\right)=c_{i}$ and we change $z^{t h}$ component of the non-participation assignment rule $p^{-i}$ then two things can happen. One possibility is that

$$
\frac{\partial c_{i}^{*}\left(p, \rho^{-i}\right)}{\partial\left(\rho^{-i}\right)^{z}}=0
$$

in that case (29), reduces to (28). Another possibility is that we move to a $c_{i}^{*}$ in the interior, in which case we are back to Case $1 .{ }^{36}$

Case 3: $c_{i}^{*}\left(p, \rho^{-i}\right)=\bar{c}_{i}$

This case is identical to the previous one.

Now, we prove the proposition.

We know that $V_{i}\left(\bar{c}_{i}\right)=\rho^{-i} \bar{\pi}_{i}^{z_{1}}\left(c_{i}^{*}\left(p, \rho^{-i}\right)\right)+\left(1-\rho^{-i}\right) \bar{\pi}_{i}^{z_{2}}\left(c_{i}^{*}\left(p, \rho^{-i}\right)\right)+\int_{c_{i}^{*}\left(p, \rho^{-i}\right)}^{\bar{c}_{i}} P_{i}(s) d s$. Because of the envelope condition proved before, that is (28), we can write

$$
\begin{aligned}
\left.\frac{d V_{i}\left(\bar{c}_{i} ; p, \rho^{-i}\right)}{d \rho^{-i}}\right|_{\rho^{-i}=p^{-i}(p)} & =\left.\frac{\partial V_{i}\left(\bar{c}_{i} ; p, \rho^{-i}\right)}{\partial \rho^{-i}}\right|_{\rho^{-i}=p^{-i}(p)} \\
& =\bar{\pi}_{i}^{z_{1}}\left(c_{i}^{*}\left(p, \rho^{-i}\right)\right)-\bar{\pi}_{i}^{z_{2}}\left(c_{i}^{*}\left(p, \rho^{-i}\right)\right) .
\end{aligned}
$$

When $\rho^{-i}$ is in a neighborhood of 0 then the outside option is flat and $c_{i}^{*}=\bar{c}_{i}$. When $\rho^{-i}$ is in a neighborhood of 1 then the outside option is very steep and $c_{i}^{*}=\underline{c}_{i}$. This means that $\left.\frac{\partial c_{i}^{*}\left(p, \rho^{-i}\right)}{\partial \rho^{-i}}\right|_{\rho^{-i}=0}=\left.\frac{\partial c_{i}^{*}\left(p, \rho^{-i}\right)}{\partial \rho^{-i}}\right|_{\rho^{-i}=1}=0$, and also we get that

$$
\begin{aligned}
\left.\frac{d V_{i}\left(\bar{c}_{i} ; p, \rho^{-i}\right)}{d \rho^{-i}}\right|_{\rho^{-i}=0} & =\bar{\pi}_{i}^{z_{1}}\left(c_{i}^{*}(p, 0)\right)-\bar{\pi}_{i}^{z_{2}}\left(c_{i}^{*}(p, 0)\right) \\
& =\bar{\pi}_{i}^{z_{1}}\left(\bar{c}_{i}\right)-\bar{\pi}_{i}^{z_{2}}\left(\bar{c}_{i}\right)<0 \\
\left.\frac{d V_{i}\left(\bar{c}_{i} ; p, \rho^{-i}\right)}{d \rho^{-i}}\right|_{\rho^{-i}=1} & =\bar{\pi}_{i}^{z_{1}}\left(c_{i}^{*}(p, 1)\right)-\bar{\pi}_{i}^{z_{2}}\left(c_{i}^{*}(p, 1)\right) \\
& =\bar{\pi}_{i}^{z_{1}}\left(\underline{c}_{i}\right)-\bar{\pi}_{i}^{z_{2}}\left(\underline{c}_{i}\right)>0 .
\end{aligned}
$$

\footnotetext{
${ }^{36}$ Note that since both $V_{i}$ and $\underline{U}_{i}$ are decreasing and convex in $c_{i}$, so changing $\left(p^{-i}\right)$ slightly cannot result in $c_{i}^{*}$ moving from $\underline{c}_{i}$ to $\bar{c}_{i}$.
} 
This implies that the optimally chosen $\rho^{-i}$, that is $p^{-i}(p)$, is interior, so it satisfies the FONC $\left.\frac{d V_{i}\left(\bar{c}_{i} ; p, \rho^{-i}\right)}{d \rho^{-i}}\right|_{\rho^{-i}=p^{-i}(p)}=0$, because of (32) it implies $\bar{\pi}_{i}^{z_{1}}\left(c_{i}^{*}\left(p, \rho^{-i}\right)\right)=\bar{\pi}_{i}^{z_{2}}\left(c_{i}^{*}\left(p, \rho^{-i}\right)\right)$. Moreover, because of the assumptions, the functions $\bar{\pi}_{i}^{z_{1}}$ and $\bar{\pi}_{i}^{z_{2}}$ cross at most once, so $c_{i}^{*}$ is uniquely determined.

We now need to verify that given $p$ there exists $p^{-i}(p)$ such that the participation constraints are satisfied. It is enough to show that $p^{-i}(p) \frac{d \bar{\pi}_{i}^{z_{1}}\left(\hat{c}_{i}\right)}{d c_{i}}+\left(1-p^{-i}(p)\right) \frac{d \bar{\pi}_{i}^{z_{2}}\left(\hat{c}_{i}\right)}{d c_{i}} \in$ $\partial V_{i}\left(\hat{c}_{i}\right)$. The first assumption of the Proposition 6 guarantees that this is always possible.

Assumption A. If for all $i$ and for all $c_{-i}$, the implication

$$
\frac{\partial J_{z_{1}}\left(c_{i}, c_{-i}\right)}{\partial c_{i}} \geq \frac{\partial J_{z_{2}}\left(c_{i}, c_{-i}\right)}{\partial c_{i}} \Longrightarrow \frac{\partial \pi_{i}^{z_{1}}\left(c_{i}, c_{-i}\right)}{\partial c_{i}} \geq \frac{\partial \pi_{i}^{z_{2}}\left(c_{i}, c_{-i}\right)}{\partial c_{i}}
$$

is satisfied, then assumption (8) is satisfied.

Proof of Sufficiency of Assumption A

If there exists a point $\left(c_{i}, c_{-i}\right)$ such that $z_{1} \in \arg \max _{z \in Z} \hat{J}_{z}\left(c_{i}^{-}, c_{-i}\right)$ and $z_{2} \in \arg \max _{z \in Z} \hat{J}_{z}\left(c_{i}^{+}, c_{-i}\right)$, then it must be the case that $\frac{\partial J_{z_{2}}\left(c_{i}, c_{-i}\right)}{\partial c_{i}} \geq \frac{\partial J_{z_{1}}\left(c_{i}, c_{-i}\right)}{\partial c_{i}}$. If Assumption A is satisfied, then we have that $\frac{\partial \pi_{i}^{z_{2}}\left(c_{i}, c_{-i}\right)}{\partial c_{i}} \geq \frac{\partial \pi_{i}^{z_{1}}\left(c_{i}, c_{-i}\right)}{\partial c_{i}}$, which by definition means that $P^{z_{2}}\left(c_{i}\right) \geq P^{z_{1}}\left(c_{i}\right)$. 


\section{Proof of Lemma 9}

We just need to prove that Assumption A is satisfied. For that, suppose that $\frac{\partial J_{z_{1}}\left(c_{i}, c_{-i}\right)}{\partial c_{i}} \geq$ $\frac{\partial J_{z_{2}}\left(c_{i}, c_{-i}\right)}{\partial c_{i}}$. By the linearity assumption, we have that $B_{i}^{z_{1}}\left[1+\left(\frac{F_{i}\left(c_{i}\right)}{f_{i}\left(c_{i}\right)}\right)^{\prime}\right] \geq B_{i}^{z_{2}}\left[1+\left(\frac{F_{i}\left(c_{i}\right)}{f_{i}\left(c_{i}\right)}\right)^{\prime}\right]$. Then, since $\left(\frac{F_{i}\left(c_{i}\right)}{f_{i}\left(c_{i}\right)}\right)^{\prime} \geq 0$ by assumption, we get $B_{i}^{z_{1}} \geq B_{i}^{z_{2}}$, which is equivalent to $\frac{\partial \pi_{i}^{z_{1}}\left(c_{i}, c_{-i}\right)}{\partial c_{i}} \geq \frac{\partial \pi_{i}^{z_{2}}\left(c_{i}, c_{-i}\right)}{\partial c_{i}}$ under the linearity assumption.

\section{Proof of Theorem 10}

The solution proposed corresponds to pointwise maximization, so the only possibility that is not optimal is that is not feasible. To check that feasibility is satisfied remember that

$$
P_{i}\left(c_{i}\right)=\int_{C_{-i}} \sum_{z \in Z} p^{z}\left(c_{i}, c_{-i}\right) \frac{\partial \pi_{i}^{z}\left(c_{i}, c_{-i}\right)}{\partial c_{i}} f_{-i}\left(c_{-i}\right) d c_{-i}
$$

and consider a fixed $c_{-i}$. In a region of cost realizations where $\bar{z} \in \arg \max _{z \in Z} \hat{J}_{z}(c), p\left(c_{i}, c_{-i}\right)$ does not change $\left(p^{\bar{z}}=1\right), P_{i}\left(c_{i}\right)$ is increasing by the convexity of $\pi_{i}^{z}\left(\cdot, c_{-i}\right)$. For a given $c_{i}^{*}$ where $z_{1} \in \arg \max _{z \in Z} \hat{J}_{z}\left(c_{i}^{*-}, c_{-i}\right)$ and $z_{2} \in \arg \max _{z \in Z} \hat{J}_{z}\left(c_{i}^{*+}, c_{-i}\right), p^{z_{1}}\left(c_{i}^{*-}, c_{-i}\right)=1$ and $p^{z_{2}}\left(c_{i}^{*+}, c_{-i}\right)=1$, so $P_{i}\left(c_{i}\right)$ is increasing because of Assumption 8 .

\section{Proof of Theorem 11}

In environments where for all $p$ at a solution of (7), (9) are satisfied, and an optimal $p^{-i}$ minimizes

$$
\underline{U}_{i}\left(c_{i}^{*}, p^{-i}\right)=E_{c_{-i}}\left[\sum_{z \in Z^{-i}}\left(p^{-i}\right)^{z}\left(c_{-i}\right) \pi_{i}^{z}\left(c_{i}^{*}, c_{-i}\right)\right],
$$

where $c_{i}^{*}$ satisfies (5) for all $p$ and $p^{-i}$. This implies that if $c_{i}^{*}$ is interior, $V_{i}$ and $\underline{U}_{i}$ must be parallel at $c_{i}^{*}$, more precisely it must be the case that $\frac{\partial \underline{U}_{i}\left(c_{i}^{*}, p^{-i}\right)}{\partial c_{i}} \in \partial V_{i}\left(c_{i}^{*}\right)$.

If we are at a corner, that is $c_{i}^{*}=\bar{c}_{i}$ or $c_{i}^{*}=\underline{c}_{i}$, then the requirement that $\frac{\partial \underline{U}_{i}\left(c_{i}^{*}, p^{-i}\right)}{\partial c_{i}} \in$ $\partial V_{i}\left(c_{i}^{*}\right)$ need not be satisfied, and the optimal $p^{-i}$ assigns probability one to the allocation that minimizes $E_{c_{-i}}\left[\pi_{i}^{z}\left(c_{i}^{*}, c_{-i}\right)\right]$ and $p^{-i}$ is independent of $p$. In the case where $c_{i}^{*} \in\left(\underline{c}_{i}, \bar{c}_{i}\right)$ the requirement that $\frac{\partial \underline{U}_{i}\left(c_{i}^{*}, p^{-i}\right)}{\partial c_{i}} \in \partial V_{i}\left(c_{i}^{*}\right)$ implies that seller will choose a different $p^{-i}(p)$ for different allocation rules $p$, even though the participation constraint will always bind at the same $c_{i}^{*}$.

\section{Proof of Corollary 12}

Let's denote by $z_{0}$ the allocation where the seller keeps all the objects and consider a fixed realization of types $c$. Since $\pi_{i}^{z_{0}}(c)$ is constant for all $i$, its derivative vanishes, and we have that $J_{z_{0}}(c)=\sum_{i=1}^{N} \pi_{i}^{z_{0}}(c)=S_{z_{0}}(c)$.

On the other hand, for every allocation $z$, its virtual surplus in the case of large externalities 
is given by

$$
J_{z}(c)=\sum_{i=1}^{N}\left[\pi_{i}^{z}(c)+\frac{\partial \pi_{i}^{z}(c)}{\partial c_{i}} \frac{F_{i}\left(c_{i}\right)-1}{f_{i}\left(c_{i}\right)}\right]>S_{z}(c)
$$

Then it is easy to see that the set where the seller keeps the objects, $\left\{c \mid z_{0} \in \arg \max _{z} S_{z}(c)\right\}$, is a subset of the set where it would be efficient that she keeps them, $\left\{c \mid z_{0} \in \arg \max _{z} J_{z}(c)\right\}$.

\section{REFERENCES}

[1] Armstrong, M. (2000): "Optimal Multi-Object Auctions," Review of Economic Studies, $67,455-181$.

[2] Avery, C. and T. Hendershott (2000): "Bundling and Optimal Auctions of Multiple Products," Review of Economic Studies, Vol. 67, No. 3.

[3] Branco, F. (1996). "Multiple Unit Auctions of an Indivisible Good," Economic Theory, vol. 8(1), pages 77-101.

[4] Brocas, Isabelle (2005): "Auctions with Type-Dependent and Negative Externalities: The Optimal Mechanism," mimeo University of Southern California.

[5] Dana, J. and K. Spier (1994): "Designing an Industry: Government Auctions with Endogenous Market Structure," Journal of Public Economics, 53, 127-147.

[6] Engelbrecht-Wiggans, R. (1988): "Revenue Equivalence in Multi-Object Auctions," Economics Letters, (26) 15-19.

[7] Figueroa, N. and V.Skreta (2005): "Optimal Multi-Unit Auctions, Who Gets What Matters," mimeo

[8] Figueroa, N. and V.Skreta (2006b): "Bunching with Multiple Objects, " mimeo

[9] Gale, I. (1990): "A multiple-object Auction with Superadditive Values," Economic Letters, 34, 323-328.

[10] Jehiel, P. and B. Moldovanu, (2001): "A Note of Revenue Maximization and Efficiency in Multi-object Auctions," Economics Bulletin, Vol. 3 no. 2, 1-5.

[11] Jehiel, P. and B. Moldovanu (2001b): "Efficient Design with Interdependent Values," Econometrica 69, 1237-1259. 
[12] Jehiel, P., B. Moldovanu and E. Stacchetti (1996): "How (Not) to sell Nuclear Weapons," American Economic Review, 86, 814-829.

[13] Jehiel, P., B. Moldovanu and E. Stacchetti (1999): "Multidimensional Mechanism Design for Auctions with Externalities," Journal of Economic Theory, 85, 258-293.

[14] Jullien, B. (2000): "Participation Constraints in Adverse Selection Models," Journal of Economic Theory, 93. 1-47.

[15] Klemperer, P. (2004): “Auctions: Theory and Practice," Princeton University Press.

[16] Krishna, V. and E. Maenner (2001): "Convex Potentials with Applications to Mechanism Design," Econometrica, 69, 1113-1119.

[17] Krishna, V. and M. Perry (2000): "Efficient Mechanism Design," mimeo Penn State University.

[18] Lewis, T. R. and D. E. M. Sappington (1989): "Countervailing Incentives in Agency Problems," Journal of Economic Theory, 49, 294-313.

[19] Maskin, E. and J. Riley (1989): "Optimal Multi-Unit Auctions," in The Economics of Missing Markets, ed. by F. Hahn, 312-335.

[20] Milgrom, P. (1996): "Procuring Universal Service: Putting Auction Theory to Work," Lecture at the Royal Academy of Sciences.

[21] Myerson, (1981): "Optimal Auction Design," Mathematics of Operations Research, 6: 58-73.

[22] Riley, J. G. and W. F. Samuelson (1981): "Optimal Auctions," American Economic Review, 71, 381-392. 

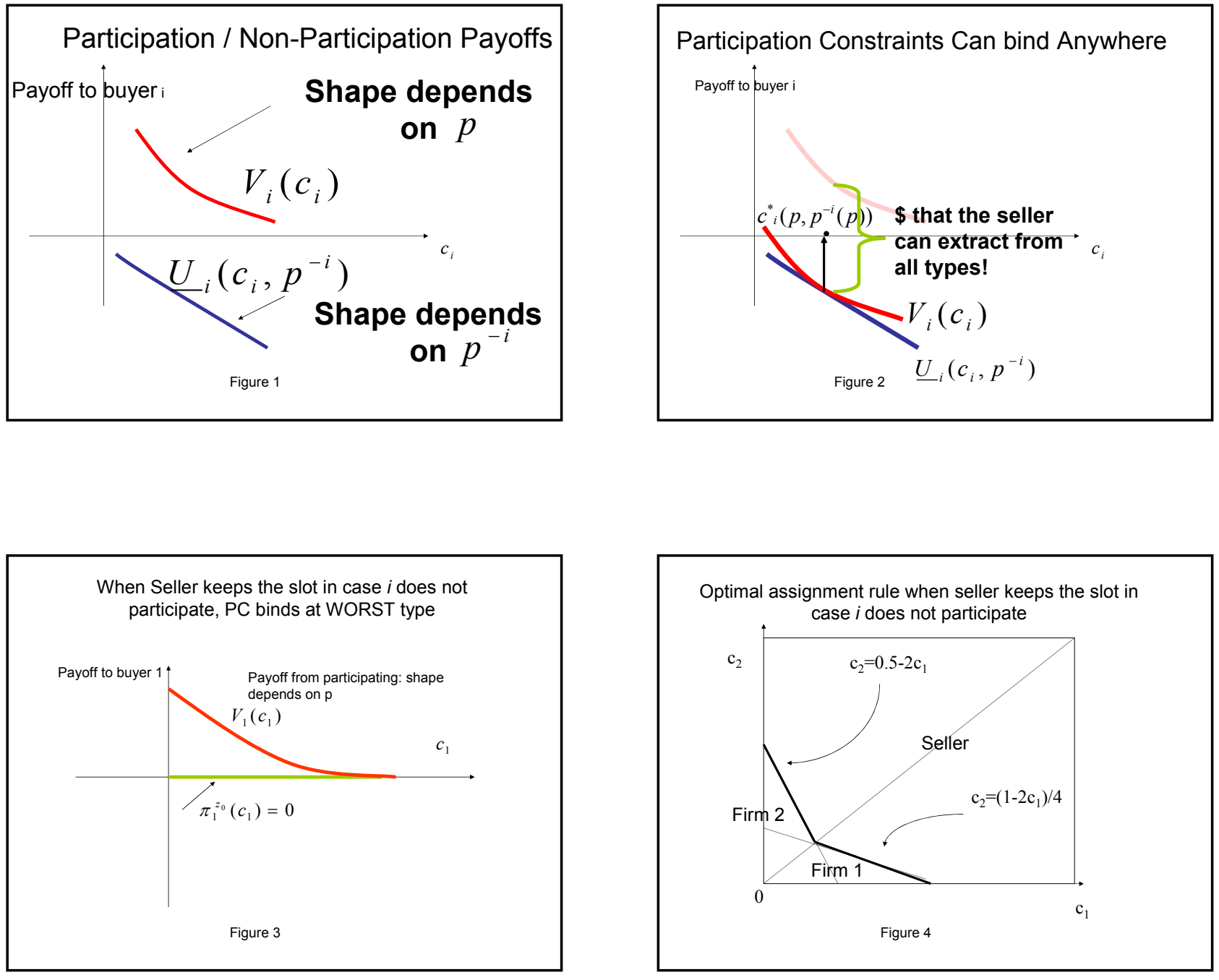

Optimal assignment rule when seller keeps the slot in

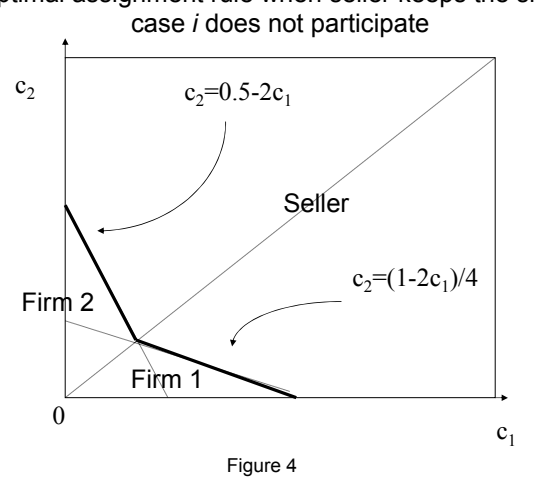

When Seller gives the slot to $j$ in case $i$ does not participate, PC binds at BEST type

Payoff to buyer 1

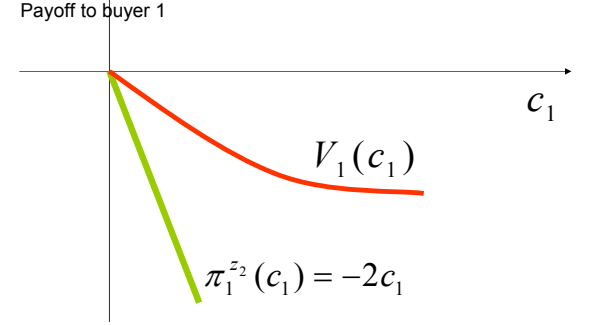

Optimal assignment rule when seller gives the slot to $j$

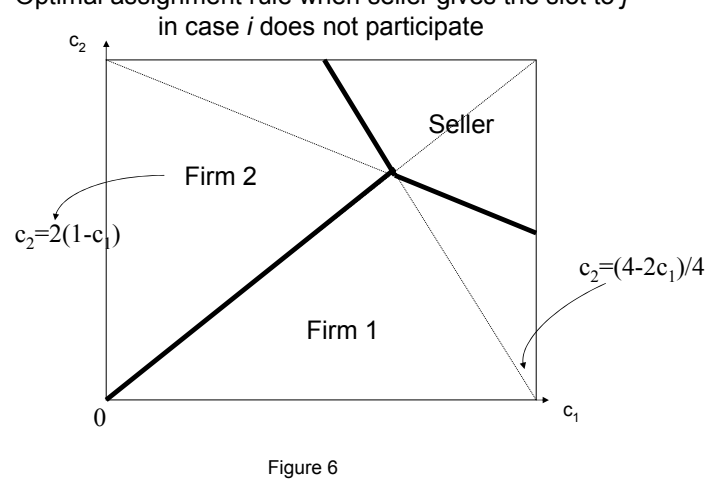



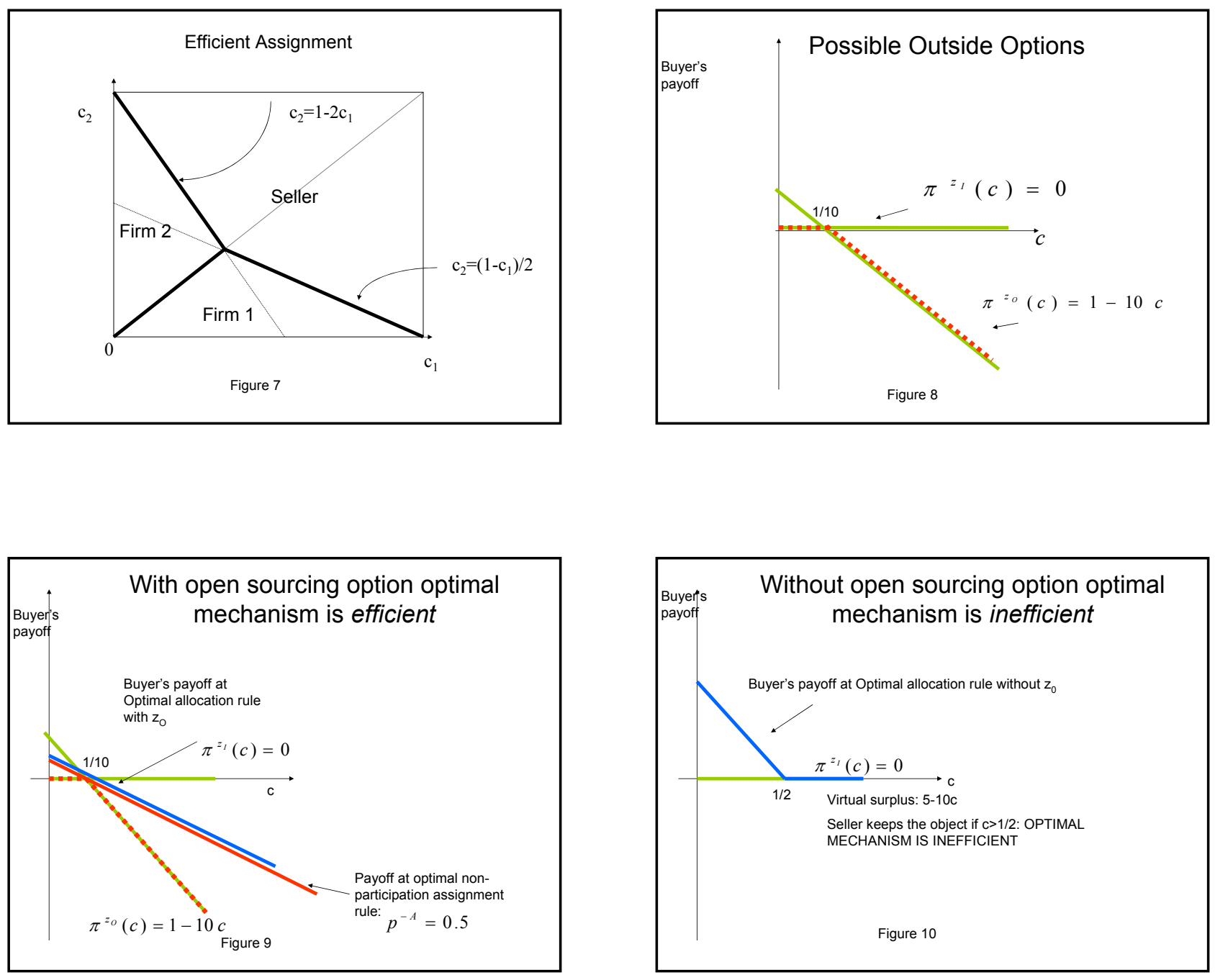